\title{
MULTIFREQUENCY ITERATIVE METHODS FOR THE INVERSE MEDIUM SCATTERING PROBLEMS IN ELASTICITY*
}

\author{
GANG $\mathrm{BAO}^{\dagger}$, TAO YIN ${ }^{\ddagger}$, AND FANG ZENG§
}

\begin{abstract}
This paper concerns the reconstruction of multiple elastic parameters (Lamé parameters and density) of an inhomogeneous medium embedded in an infinite homogeneous isotropic background in $\mathbb{R}^{2}$. The direct scattering problem is reduced to an equivalent system on a bounded domain by introducing an exact transparent boundary condition and the well-posedness of the corresponding variational problem is established. The Fréchet differentiability of the near-field scattering map is studied with respect to the elastic parameters. Based on the multifrequency measurement data and its phaseless term, two Landweber iterative algorithms are developed for the reconstruction of the multiple elastic parameters. Numerical examples, indicating that plane pressure incident wave is a better choice, are presented to show the validity and accuracy of our methods.
\end{abstract}

Key words. elastic wave, inverse medium problem, iterative method, multifrequency

AMS subject classifications. 35J25, 35R30, 65N21, 74B05

DOI. $10.1137 / 18 \mathrm{M} 1220844$

1. Introduction. Time-harmonic elastic scattering problems play important roles in many fields of applications, and the linear elasticity theory provides an essential tool for analysis and design of mechanical systems and engineering structures $[3,20]$. In this paper, we consider several inhomogeneous isotropic elastic bodies embedded in an infinite homogeneous isotropic background medium in $\mathbb{R}^{2}$. Denote by $\lambda, \mu$ the Lamé parameters with $\mu>0, \lambda>0$, and by $\rho$ the density of the elastic medium. Suppose that $\lambda=\lambda_{0}\left(1+q_{\lambda}\right), \mu=\mu_{0}\left(1+q_{\mu}\right)$, and $\rho=\rho_{0}\left(1+q_{\rho}\right)$, where $\lambda_{0}, \mu_{0}$, and $\rho_{0}$ are constants representing the Lamé parameters and density of the background elastic medium. Denote $B_{R}:=\left\{x \in \mathbb{R}^{2}:|x|<R\right\}$ and $\Gamma_{R}:=\partial B_{R}$. Set $q:=\left(q_{\lambda}, q_{\mu}, q_{\rho}\right)^{\top}$. Throughout, we make the following assumption.

Assumption: There exist some $R>0$ and constants $C_{1}, C_{2}>0$ such that

$$
\operatorname{supp}\{q\} \subset B_{R}, q \in \mathcal{K}:=\left\{q \in L^{\infty}\left(B_{R}\right)^{3}:-1<C_{1} \leq q_{\lambda}, q_{\mu}, q_{\rho} \leq C_{2}<\infty\right\} .
$$

Let $u^{\text {in }}$ be a plane incident field satisfying

$$
\nabla \cdot \sigma_{0,0}\left(u^{i n}\right)+\rho_{0} \omega^{2} u^{i n}=0 \quad \text { in } \quad \mathbb{R}^{2},
$$

*Submitted to the journal's Computational Methods in Science and Engineering section October 15, 2018; accepted for publication (in revised form) April 30, 2019; published electronically July 16, 2019.

https://doi.org/10.1137/18M1220844

Funding: The work of the first author was partially supported by an NSFC Innovative Group Fund (11621101), an Integrated Project of the Major Research Plan of NSFC (91630309), and an NSFC A3 Project (11421110002). The work of the third author was supported by NSFC grants (11501063, 11771068), the Chongqing Research Program of Basic Research and Frontier Technology (CSTC2017JCYJAX0294), and the Fundamental Research Funds for the Central University (106112016CDJXY100004).

†School of Mathematical Sciences, Zhejiang University, Hangzhou 310027, China (baog@zju.edu.cn).

${ }^{\ddagger}$ Department of Computing \& Mathematical Sciences, California Institute of Technology, Pasadena, CA 91125 (taoyin89@caltech.edu).

$\S$ College of Mathematics and Statistics, Chongqing University, Chongqing 400044, China (fzeng@cqu.edu.cn).

B721

Copyright $@$ by SIAM. Unauthorized reproduction of this article is prohibited. 
where $\omega>0$ is the frequency and the stress tensor $\sigma_{q_{\lambda}, q_{\mu}}(u)$ is defined as

$$
\sigma_{q_{\lambda}, q_{\mu}}(u):=\lambda_{0}\left(1+q_{\lambda}\right)(\operatorname{div} u) \mathbf{I}+2 \mu_{0}\left(1+q_{\mu}\right) \mathcal{E}(u), \quad \mathcal{E}(u):=\frac{1}{2}\left(\nabla u+\nabla u^{\top}\right),
$$

and $\mathbf{I}$ stands for the $2 \times 2$ identity matrix. The elliptic equation (1.1) can be restated as

$$
\Delta_{\lambda_{0}, \mu_{0}}^{*} u^{i n}+\rho_{0} \omega^{2} u^{i n}=0 \text { in } \mathbb{R}^{2},
$$

where the Lamé operator $\Delta_{\lambda, \mu}^{*}$ is defined as

$$
\Delta_{\lambda, \mu}^{*}=\mu \operatorname{div} \operatorname{grad}+(\lambda+\mu) \operatorname{grad} \operatorname{div} .
$$

In this paper, the incident wave is allowed to be either a plane shear wave taking the form

$$
u^{i n}=u_{s}^{i n}:=d^{\perp} e^{i k_{s} x \cdot d}, \quad d=\left(\cos \theta^{i n}, \sin \theta^{i n}\right)^{\top} \in \Gamma_{1}, d^{\perp}=\left(-\sin \theta^{i n}, \cos \theta^{i n}\right)^{\top},
$$

or a plane pressure wave taking the form

$$
u^{i n}=u_{p}^{i n}:=d e^{i k_{p} x \cdot d}, \quad d \in \Gamma_{1},
$$

where

$$
k_{s}=\omega \sqrt{\frac{\rho_{0}}{\mu_{0}}}, \quad k_{p}=\omega \sqrt{\frac{\rho_{0}}{\lambda_{0}+2 \mu_{0}}}
$$

are the wave numbers of the pressure wave and shear wave, respectively, and $d$ and $\theta^{i n}$ are referred to as the direction and angle of the incidence, respectively.

The total displacement field $u=\left(u_{1}, u_{2}\right)^{\top}$ can be modeled by the reduced Navier equation

$$
\nabla \cdot \sigma_{q_{\lambda}, q_{\mu}}(u)+\omega^{2} \rho u=0 \quad \text { in } \quad \mathbb{R}^{2} .
$$

Since the background medium is unbounded, an appropriate radiation condition at infinity must be imposed on the scattered field $u^{s c}:=u-u^{\text {in }}$ to ensure well-posedness of the scattering problem. The scattered field in $\mathbb{R}^{2} \backslash \overline{B_{R}}$ can be decomposed into the sum of the compressional (longitudinal) part $u_{p}^{s c}$ and the shear (transversal) part $u_{s}^{s c}$ as follows:

$$
u^{s c}=u_{p}^{s c}+u_{s}^{s c}, \quad u_{p}^{s c}=-\frac{1}{k_{p}^{2}} \operatorname{grad} \operatorname{div} u^{s c}, \quad u_{s}^{s c}=\frac{1}{k_{s}^{2}} \overrightarrow{\operatorname{curl}} \operatorname{curl} u^{s c},
$$

where the two-dimensional operators curl and $\overrightarrow{c u r l}$ are defined respectively by

$$
\operatorname{curl} v=\partial_{1} v_{2}-\partial_{2} v_{1}, \quad v=\left(v_{1}, v_{2}\right)^{\top}, \quad \overrightarrow{\operatorname{curl}} f:=\left(\partial_{2} f,-\partial_{1} f\right)^{\top} .
$$

It then follows from the decompositions in (1.3) that

$$
\left(\Delta+k_{\alpha}^{2}\right) u_{\alpha}^{s c}=0, \quad \alpha=p, s, \quad \operatorname{div} u_{s}^{s c}=0, \quad \operatorname{curl} u_{p}^{s c}=0 .
$$

The scattered field is required to satisfy the Kupradze radiation condition (see, e.g., [30])

$$
\lim _{r \rightarrow \infty} r^{\frac{1}{2}}\left(\frac{\partial u_{t}^{s c}}{\partial r}-i k_{t} u_{t}^{s c}\right)=0, \quad r=|x|, \quad t=p, s
$$

Copyright (c) by SIAM. Unauthorized reproduction of this article is prohibited. 
uniformly with respect to all $\hat{x}=x /|x| \in \Gamma_{1}$.

Given the incident field $u^{\text {in }}$, the direct problem is to determine the scattered field $u^{s c}$ for the known elastic parameters $\lambda, \mu, \rho$. In practice, the original boundary value problem for the Lamé system can be reduced to an equivalent system on a bounded domain via introducing an exact transparent boundary condition (TBC) on an artificial boundary enclosing the inhomogeneous bodies. The TBC can be formulated by the so-called Dirichlet-to-Neumann ( $\mathrm{DtN})$ map taking the form of a Fourier series [6, 22, 31, 33]. Based on the properties of the DtN map and the Fredholm alternative theorem, the uniqueness and existence of weak solutions of the equivalent system can be derived (section 2, Theorem 2.4).

The main purpose of this paper is to study the numerical algorithm for the inverse medium problem in elastic scattering, that is, to determine the unknown elastic parameters $q_{\lambda}, q_{\mu}, q_{\rho}$ from the measurements of near-field data $\left.u\right|_{\Gamma_{R}}$, given the incident field $u^{i n}$. For the static case, i.e., $\omega=0$, uniqueness of the inverse medium problem has been investigated under appropriate assumptions on the elastic parameters in $[1,18,21,27,34,36,35]$. For the time-harmonic case, Beretta et al. [17] prove uniqueness when the Lamé parameters and the density are assumed to be piecewise constant on a given domain partition. For the mathematical analysis of the stability for the inverse medium problems in elasticity, we refer the reader to $[2,18,26,34,35,36]$.

Recently, it has been realized that the use of multifrequency data is an effective approach to overcoming the major difficulties associated with the inverse medium problems: the ill-posedness and the presence of many local minima. Based on the multifrequency measurements and the Fréchet derivative of the solution operator, a stable recursive linearization method is proposed in [7] for the inverse medium problems in acoustics; see also $[8,9,10,11]$. The idea to use multifrequency data has also been widely developed in proving uniqueness and increasing stability for inverse source problems in acoustics, elastodynamics, and electromagnetics $[5,12,13$, $14,15,19,25,28,32,33]$. It still remains open whether or not the multifrequency measurements uniquely determine the Lamé parameters and the density. This paper is designed to study the capability of iterative methods for inverse medium problems in elasticity using multifrequency measurements. Compared with the acoustic and electromagnetic case, the elasticity problem appears to be more complicated because of the coexistence of pressure and shear waves that propagate at different speeds and the aim to reconstruct multiple parameters. In particular, if the Lamé parameters are constants, the inverse medium problem in elasticity is consistent with that in acoustics; see section 4 . Relying on the variational arguments for the direct problems, we derive the Fréchet derivative of the solution operator with respect to the elastic parameters and investigate the adjoint of the Fréchet derivative. Then we employ the Landweber iterative method based on the multifrequency measurements to find the unknown elastic parameters. At each iteration step, the forward problem and an adjoint one need to be solved and the correctness of the parameters needs to be evaluated.

The outline of the paper is as follows. In section 2, we derive the well-posedness of the direct scattering problem using the variational approach and investigate the Fréchet differentiability of the near-field scattering map. We develop the Landweber iterative methods for solving the inverse medium problem in section 3 . In section 4 , we give a brief discussion about a special case when $q_{\lambda}=q_{\mu}=0$. Numerical examples are presented in section 5 . 
2. Direct scattering problem. In this section, we discuss the well-posedness of the direct elastic scattering problem and investigate the Fréchet differentiability of the near-field scattering map.

2.1. Variational formulation. We first reduce the original scattering problem described in section 1 on a bounded domain via introducing a TBC on an artificial boundary $\Gamma_{R}$ enclosing the inhomogeneity inside. The TBC is formulated by the so-called DtN map defined as follows.

Definition 2.1. For any $w \in H^{1 / 2}\left(\Gamma_{R}\right)^{2}$, The DtN map $\mathcal{B}$ applied to $w$ is defined as $\left.T_{\lambda_{0}, \mu_{0}} v^{s c}\right|_{\Gamma_{R}}$, where $v^{s c}$ satisfies

$$
\begin{aligned}
\Delta_{\lambda_{0}, \mu_{0}}^{*} v^{s c}+\rho_{0} \omega^{2} v^{s c} & =0 \quad \text { in } \quad \mathbb{R}^{2} \backslash \overline{B_{R}}, \\
v^{s c} & =w \quad \text { on } \quad \Gamma_{R},
\end{aligned}
$$

and the Kupradze radiation condition. Here, $T_{\lambda_{0}, \mu_{0}}$ is the traction operator defined by

$$
T_{\lambda_{0}, \mu_{0}} u:=\nu \cdot \sigma_{0,0}(u)=2 \mu_{0} \partial_{\nu} u+\lambda_{0} \nu \operatorname{div} u-\mu_{0} \nu^{\perp} \operatorname{curl} \mathrm{u},
$$

where $\nu=\left(\nu_{1}, \nu_{2}\right)^{\top}$ denotes the exterior unit normal vector to $\Gamma_{R}$ and the corresponding tangential vector is given by $\nu^{\perp}:=\left(-\nu_{2}, \nu_{1}\right)^{\top}$.

The DtN map $\mathcal{B}$ is well defined since the Dirichlet-type boundary value problem (2.1)-(2.2) is uniquely solvable in $H_{l o c}^{1}\left(\mathbb{R}^{2} \backslash \overline{B_{R}}\right)^{2}$; see Corollary 2.3 in [6]. For all $n \in \mathbb{Z}$, denote

$$
\alpha_{n}\left(t_{\xi}\right):=\frac{H_{n}^{(1)^{\prime}}\left(t_{\xi}\right)}{H_{n}^{(1)}\left(t_{\xi}\right)}, \quad \beta_{n}\left(t_{\xi}\right):=\frac{H_{n}^{(1)^{\prime \prime}}\left(t_{\xi}\right)}{H_{n}^{(1)}\left(t_{\xi}\right)}, \quad \xi=p, s,
$$

where

$$
t_{\xi}=k_{\xi} R
$$

Following the procedure described in [6], it can be derived that

$$
\mathcal{B} w=\sum_{n \in \mathbb{Z}} \frac{1}{2 \pi R} M_{\theta}^{\top} W_{n} \int_{0}^{2 \pi} M_{\phi} w(R, \phi) e^{i n(\theta-\phi)} d \phi,
$$

where the matrix $M_{\theta}$ dependent on the angle $\theta \in[0,2 \pi)$ is defined as

$$
M_{\theta}:=\left[\begin{array}{cc}
\cos \theta & \sin \theta \\
-\sin \theta & \cos \theta
\end{array}\right]
$$

and the coefficient matrix $W_{n}$ is given by

$$
W_{n}=B_{n} A_{n}^{-1},
$$

with

$$
\begin{aligned}
A_{n} & :=\left[\begin{array}{cc}
t_{p} \alpha_{n}\left(t_{p}\right) & \text { in } \\
i n & -t_{s} \alpha_{n}\left(t_{s}\right)
\end{array}\right] \\
B_{n} & :=\left[\begin{array}{cc}
2 \mu_{0} t_{p}^{2} \beta_{n}\left(t_{p}\right)-\lambda_{0} t_{p}^{2} & 2 i \mu_{0} n\left(t_{s} \alpha_{n}\left(t_{s}\right)-1\right) \\
2 i \mu_{0} n\left(t_{p} \alpha_{n}\left(t_{p}\right)-1\right) & -2 \mu_{0} t_{s}^{2} \beta_{n}\left(t_{s}\right)-\mu_{0} t_{s}^{2}
\end{array}\right] .
\end{aligned}
$$

For the invertibility of matrix $A_{n}$, we refer the reader to Lemma 2.11 in [6]. Denote by $\Lambda_{n}$ the determinant of $A_{n}$. Moreover, it can be proved that $\mathcal{B}$ is a bounded linear operator from $H^{1 / 2}\left(\Gamma_{R}\right)^{2}$ to $H^{-1 / 2}\left(\Gamma_{R}\right)^{2}$; see Lemma 2.13(i) in [6]. 
Lemma 2.2. For all $\varphi \in\left(H^{1 / 2}\left(\Gamma_{R}\right)\right)^{2}$, the DtN mapping $\mathcal{B}$ can be expressed equivalently as

$$
\mathcal{B} \varphi:=\sum_{n \in \mathbb{Z}} \frac{1}{2 \pi R} M_{\theta}^{\top}\left[W_{n}\right]^{\top} \int_{0}^{2 \pi} M_{\phi} \varphi e^{i n(\phi-\theta)} d \phi .
$$

In addition, it holds that

$$
\mathcal{B}^{*} \varphi=\overline{\mathcal{B}} \bar{\varphi} .
$$

Proof. We first prove (2.6). We can derive that for all $\varphi, \psi \in\left(H^{1 / 2}\left(\Gamma_{R}\right)\right)^{2}$,

$$
\begin{aligned}
\left\langle\mathcal{B}^{*} \varphi, \psi\right\rangle_{\Gamma_{R}} & =\langle\varphi, \mathcal{B} \psi\rangle_{\Gamma_{R}} \\
& =\int_{0}^{2 \pi} \sum_{n \in \mathbb{Z}} \frac{1}{2 \pi} \varphi^{\top} M_{\theta}^{\top} \overline{W_{n}} \int_{0}^{2 \pi} M_{\phi} \bar{\psi} e^{i n(\phi-\theta)} d \phi d \theta \\
& =\int_{0}^{2 \pi} \sum_{n \in \mathbb{Z}} \frac{1}{2 \pi} \bar{\psi}^{\top} M_{\phi}^{\top} \overline{\left[W_{n}\right]^{\top}} \int_{0}^{2 \pi} M_{\theta} \varphi e^{i n(\phi-\theta)} d \theta d \phi \\
& =\int_{0}^{2 \pi} \sum_{n \in \mathbb{Z}} \frac{1}{2 \pi} \bar{\psi}^{\top} \overline{\left\{M_{\phi}^{\top}\left[W_{n}\right]^{\top} \int_{0}^{2 \pi} M_{\theta} \bar{\varphi} e^{i n(\theta-\phi)} d \theta\right\}} d \phi \\
& =\langle\overline{\mathcal{B} \bar{\varphi}}, \psi\rangle_{\Gamma_{R}},
\end{aligned}
$$

where $\langle\cdot, \cdot\rangle_{\Gamma_{R}}$ is the $L^{2}$ duality pairing between $\left(H^{-1 / 2}\left(\Gamma_{R}\right)\right)^{2}$ and $\left(H^{1 / 2}\left(\Gamma_{R}\right)\right)^{2}$. It remains to prove (2.5). It follows from (2.3) that

$$
\begin{aligned}
\mathcal{B} w & =\sum_{n \in \mathbb{Z}} \frac{1}{2 \pi R} M_{\theta}^{\top} W_{n} \int_{0}^{2 \pi} M_{\phi} w(R, \phi) e^{i n(\theta-\phi)} d \phi \\
& =\sum_{n \in \mathbb{Z}} \frac{1}{2 \pi R} M_{\theta}^{\top} W_{-n} \int_{0}^{2 \pi} M_{\phi} w(R, \phi) e^{i n(\phi-\theta)} d \phi,
\end{aligned}
$$

where

$$
W_{-n}=B_{-n} A_{-n}^{-1}
$$

with

$$
\begin{aligned}
& A_{-n}:=\left[\begin{array}{cc}
t_{p} \alpha_{n}\left(t_{p}\right) & -i n \\
-i n & -t_{s} \alpha_{n}\left(t_{s}\right)
\end{array}\right], \\
& B_{-n}:=\left[\begin{array}{cc}
2 \mu_{0} t_{p}^{2} \beta_{n}\left(t_{p}\right)-\lambda_{0} t_{p}^{2} & -2 i \mu_{0} n\left(t_{s} \alpha_{n}\left(t_{s}\right)-1\right) \\
-2 i \mu_{0} n\left(t_{p} \alpha_{n}\left(t_{p}\right)-1\right) & -2 \mu_{0} t_{s}^{2} \beta_{n}\left(t_{s}\right)-\mu_{0} t_{s}^{2}
\end{array}\right] .
\end{aligned}
$$

It follows from the properties of the Hankel function that

$$
H_{n}^{(1)^{\prime \prime}}(z)=\left(\frac{n^{2}}{z^{2}}-1\right) H_{n}^{(1)}(z)-\frac{1}{z} H_{n}^{(1)^{\prime}}(z),
$$

giving rise to the identities

$$
\beta_{n}\left(t_{p}\right)=\frac{n^{2}}{t_{p}^{2}}-1-\frac{1}{t_{p}} \alpha_{n}\left(t_{p}\right), \quad \beta_{n}\left(t_{s}\right)=\frac{n^{2}}{t_{s}^{2}}-1-\frac{1}{t_{s}} \alpha_{n}\left(t_{s}\right) .
$$

Copyright (c) by SIAM. Unauthorized reproduction of this article is prohibited. 
From the expressions of $A_{n}^{-1}$ and $B_{n}$ we get the entries $W_{n}^{(i, j)}$ of $W_{n}$, given by (see also Lemma 2.13 in [6])

$$
\begin{aligned}
W_{n}^{(1,1)} & =\frac{1}{R \Lambda_{n}}\left[-2 \mu \Lambda_{n}+\rho_{0} \omega^{2} R^{2} t_{s} \alpha_{n}\left(t_{s}\right)\right], \\
W_{n}^{(2,2)} & =\frac{1}{R \Lambda_{n}}\left[-2 \mu \Lambda_{n}+\rho_{0} \omega^{2} R^{2} t_{p} \alpha_{n}\left(t_{p}\right)\right], \\
W_{n}^{(1,2)} & =\frac{1}{R \Lambda_{n}}\left[-2 i n \mu \Lambda_{n}+i n \rho_{0} \omega^{2} R^{2}\right] \\
W_{n}^{(2,1)} & =-W_{n}^{(1,2)} .
\end{aligned}
$$

Then we can obtain that $W_{-n}^{(1,1)}=W_{n}^{(1,1)}, W_{-n}^{(2,2)}=W_{n}^{(2,2)}$, and $W_{-n}^{(1,2)}=W_{n}^{(2,1)}$. These further imply that $W_{-n}=\left[W_{n}\right]^{\top}$, which completes the proof.

The following property of the coefficient matrix $W_{n}$ can be obtained directly from Lemma 2.13 in [6].

Lemma 2.3. The matrix $\widetilde{W}_{n}=-\left(W_{n}+W_{n}^{*}\right) / 2$ is positive definite for sufficiently large $|n|$.

Using the DtN map, we can impose the following TBC for the scattered field:

$$
T_{\lambda_{0}, \mu_{0}} u^{s c}=\mathcal{B} u^{s c} \quad \text { on } \quad \Gamma_{R}
$$

Note that

$$
T_{\lambda_{0}, \mu_{0}} u=\mathcal{B} u+T_{\lambda_{0}, \mu_{0}} u^{i n}-\mathcal{B} u^{i n} \quad \text { on } \quad \Gamma_{R} .
$$

Then the original scattering problem is equivalently reduced to the following nonlocal boundary value problem:

$$
\begin{aligned}
\nabla \cdot \sigma_{q_{\lambda}, q_{\mu}}(u)+\rho \omega^{2} u=0 & \text { in } \quad B_{R}, \\
T_{\lambda_{0}, \mu_{0}} u-\mathcal{B} u-g=0 & \text { on } \quad \Gamma_{R},
\end{aligned}
$$

where $g:=T_{\lambda_{0}, \mu_{0}} u^{i n}-\mathcal{B} u^{i n}$. Then the variational formulation of (2.7)-(2.8) reads as follows: find $u=\left(u_{1}, u_{2}\right)^{\top} \in\left(H^{1}\left(B_{R}\right)\right)^{2}$ such that

$(2.9) a_{q}(u, v)-\int_{\Gamma_{R}} \mathcal{B} u \cdot \bar{v} d s=\int_{\Gamma_{R}} g \cdot \bar{v} d s \quad$ for all $\quad v=\left(v_{1}, v_{2}\right)^{\top} \in\left(H^{1}\left(B_{R}\right)\right)^{2}$,

where the sesquilinear form $a_{q}(\cdot, \cdot):\left(H^{1}\left(B_{R}\right)\right)^{2} \times\left(H^{1}\left(B_{R}\right)\right)^{2} \rightarrow \mathbb{C}$ is defined by

$$
a_{q}(u, v):=A_{q_{\lambda}}(u, v)+B_{q_{\mu}}(u, v)+C_{q_{\rho}}(u, v)
$$

and

$$
\begin{aligned}
& A_{q_{\lambda}}(u, v)=\int_{B_{R}} \lambda_{0}\left(1+q_{\lambda}\right)(\nabla \cdot u)(\nabla \cdot \bar{v}) d x, \\
& B_{q_{\mu}}(u, v)=2 \int_{B_{R}} \mu_{0}\left(1+q_{\mu}\right) \mathcal{E}(u): \mathcal{E}(\bar{v}) d x, \\
& C_{q_{\rho}}(u, v)=-\int_{B_{R}} \rho_{0}\left(1+q_{\rho}\right) \omega^{2} u \cdot \bar{v} d x .
\end{aligned}
$$

Copyright $@$ ㅇ by SIAM. Unauthorized reproduction of this article is prohibited. 
The double dot notation appearing in $B_{q_{\mu}}$ is understood in the following way. If tensors $\mathbf{A}$ and $\mathbf{B}$ have rectangular Cartesian components $a_{i j}$ and $b_{i j}, i, j=1,2$, respectively, then the double contraction of $\mathbf{A}$ and $\mathbf{B}$ is

$$
\mathbf{A}: \mathbf{B}=\sum_{i=1}^{2} \sum_{j=1}^{2} a_{i j} b_{i j}
$$

The well-posedness of the variational formulation (2.9) is a consequence of the following theorem.

Theorem 2.4. For any $f \in L^{2}\left(B_{R}\right)^{2}$ and $g \in H^{-1 / 2}\left(\Gamma_{R}\right)^{2}$, there exists a unique weak solution $u \in H^{1}\left(B_{R}\right)^{2}$ to the boundary value problem

$$
\begin{array}{rcc}
\nabla \cdot \sigma_{q_{\lambda}, q_{\mu}}(u)+\rho \omega^{2} u=f & \text { in } \quad B_{R}, \\
T u-\mathcal{B} u-g=0 \quad \text { on } & \Gamma_{R},
\end{array}
$$

and

$$
\|u\|_{H^{1}\left(B_{R}\right)^{2}} \leq \gamma_{q}^{-1}\left(\|f\|_{L^{2}\left(B_{R}\right)^{2}}+\|g\|_{H^{-1 / 2}\left(\Gamma_{R}\right)^{2}}\right),
$$

where $\gamma_{q}>c_{0}>0$ is a constant independent of $u$.

Proof. We know from Lemma 2.3 that $-\operatorname{Re}\left(W_{n}\right)$ is positive definite for large $|n|$. The operator $-\mathcal{B}$ can be decomposed into the sum of an operator $\mathcal{B}_{1}$ whose real part is positive definite and a finite rank operator $\mathcal{B}_{2}$ from $H^{1 / 2}\left(\Gamma_{R}\right)^{2}$ to $H^{-1 / 2}\left(\Gamma_{R}\right)^{2}$. Define the sesquilinear form $A(\cdot, \cdot): H^{1}\left(B_{R}\right)^{2} \times H^{1}\left(B_{R}\right)^{2} \rightarrow \mathbb{C}$ as

$$
A(u, v)=a_{q}(u, v)-\int_{\Gamma_{R}} \mathcal{B} u \cdot \bar{v} d s .
$$

Then the variational equation corresponding to (2.11)-(2.12) reads

$$
A(u, v)=\int_{\Gamma_{R}} g \cdot \bar{v} d s-\int_{B_{R}} f \cdot \bar{v} d s \quad \text { for all } \quad v \in H^{1}\left(B_{R}\right)^{2} .
$$

We split the sequilinear form $A$ into the sum $A=A_{1}+A_{2}$, where

$$
\begin{aligned}
A_{1}(u, v)= & \int_{B_{R}}\left[\lambda_{0}\left(1+q_{\lambda}\right)(\nabla \cdot u)(\nabla \cdot \bar{v})+2 \mu_{0}\left(1+q_{\mu}\right) \mathcal{E}(u): \mathcal{E}(\bar{v})+u \cdot \bar{v}\right] d x \\
& +\int_{\Gamma_{R}} \mathcal{B}_{1} u \cdot \bar{v} d s \\
A_{2}(u, v)= & -\int_{B_{R}}\left[\rho_{0}\left(1+q_{\rho}\right) \omega^{2}+1\right] u \cdot \bar{v} d x+\int_{\Gamma_{R}} \mathcal{B}_{2} u \cdot \bar{v} d s
\end{aligned}
$$

Recalling the Korn inequality (see, e.g., [24]), we have

$$
\operatorname{Re} A_{1}(v, v) \geq c_{1}\|v\|_{H^{1}\left(B_{R}\right)^{2}}^{2} \text { for all } \quad v \in H^{1}\left(B_{R}\right)^{2},
$$

with some constant $c_{1}>0$. Moreover, applying the Cauchy-Schwarz inequality yields

$$
\operatorname{Re} A_{2}(v, v) \geq-c_{2}\|v\|_{L^{2}\left(B_{R}\right)^{2}}^{2}+\operatorname{Re}\left\langle\mathcal{B}_{2} v, v\right\rangle_{\Gamma_{R}} \text { for all } v \in H^{1}\left(B_{R}\right)^{2},
$$

for some constant $c_{2}>0$. From the compact embedding $H^{1}\left(B_{R}\right) \hookrightarrow L^{2}\left(B_{R}\right)$ and the compactness of $\mathcal{B}_{2}$, we conclude that the sesquilinear form $A$ is strongly elliptic

Copyright $@$ by SIAM. Unauthorized reproduction of this article is prohibited. 
(see Definition 2.5) over $H^{1}\left(B_{R}\right)^{2} \times H^{1}\left(B_{R}\right)^{2}$. The sesquilinear form $A$ obviously generates a continuous linear operator $\mathcal{A}: H^{1}\left(B_{R}\right)^{2} \rightarrow\left(H^{1}\left(B_{R}\right)^{2}\right)^{\prime}$ such that

$$
A(u, v)=\langle\mathcal{A} u, v\rangle \quad \text { for all } \quad v \in H^{1}\left(B_{R}\right)^{2} .
$$

Here $\left(H^{1}\left(B_{R}\right)^{2}\right)^{\prime}$ denotes the dual space of $H^{1}\left(B_{R}\right)^{2}$ with respect to the duality $\langle\cdot, \cdot\rangle$ extending the $L^{2}$ scalar product in $L^{2}\left(B_{R}\right)^{2}$. We know from Rellich's lemma in elasticity (see Lemma 2.14 in [6]) that the homogeneous operator equation $\mathcal{A} u=0$ has only the trivial solution $u=0$. Then it follows from the Fredholm alternative that the variational formulation (2.9) is uniquely solvable. Finally, the inf-sup condition

$$
\sup _{0 \neq v \in\left(H^{1}\left(B_{R}\right)\right)^{2}} \frac{|A(u, v)|}{\|v\|_{H^{1}\left(B_{R}\right)^{2}}} \geq \gamma_{q}\|u\|_{H^{1}\left(B_{R}\right)^{2}} \quad \text { for all } \quad u \in H^{1}\left(B_{R}\right)^{2},
$$

with some constant $\gamma_{q}>0$ generated from the general theory in Babuška and Aziz [4], implies the estimate (2.13). In fact, for $F \in\left(H^{1}\left(B_{R}\right)^{2}\right)^{\prime}$, considering the operator equation $\mathcal{A} u=F$ related to the variational equation (2.14), it follows from the Babuška theory that the operator equation is well-posed if and only if the conditions

$$
\inf _{0 \neq u \in\left(H^{1}\left(B_{R}\right)\right)^{2}} \sup _{0 \neq v \in\left(H^{1}\left(B_{R}\right)\right)^{2}} \frac{|A(u, v)|}{\|u\|_{H^{1}\left(B_{R}\right)^{2}}\|v\|_{H^{1}\left(B_{R}\right)^{2}}}=C_{U}>0
$$

and

$$
\inf _{0 \neq v \in\left(H^{1}\left(B_{R}\right)\right)^{2}} \sup _{0 \neq u \in\left(H^{1}\left(B_{R}\right)\right)^{2}} \frac{|A(u, v)|}{\|u\|_{H^{1}\left(B_{R}\right)^{2}}\|v\|_{H^{1}\left(B_{R}\right)^{2}}}=C_{E}>0
$$

hold and they are equivalent to the uniqueness and existence of solution of the operator equation, respectively. Then it follows from the variational equation (2.14) and the trace theorem that

$$
\begin{aligned}
\gamma_{q}\|u\|_{H^{1}\left(B_{R}\right)^{2}} & \leq \sup _{0 \neq v \in\left(H^{1}\left(B_{R}\right)\right)^{2}} \frac{|A(u, v)|}{\|v\|_{H^{1}\left(B_{R}\right)^{2}}} \\
& \leq \sup _{0 \neq v \in\left(H^{1}\left(B_{R}\right)\right)^{2}} \frac{\|g\|_{H^{-1 / 2}\left(\Gamma_{R}\right)^{2}}\|v\|_{H^{1 / 2}\left(\Gamma_{R}\right)^{2}}+\|f\|_{L^{2}\left(B_{R}\right)^{2}}\|v\|_{L^{2}\left(B_{R}\right)^{2}}}{\|v\|_{H^{1}\left(B_{R}\right)^{2}}} \\
& \leq \sup _{0 \neq v \in\left(H^{1}\left(B_{R}\right)\right)^{2}} \frac{\|g\|_{H^{-1 / 2}\left(\Gamma_{R}\right)^{2}}\|v\|_{H^{1}\left(B_{R}\right)^{2}}+\|f\|_{L^{2}\left(B_{R}\right)^{2}}\|v\|_{H^{1}\left(B_{R}\right)^{2}}}{\|v\|_{H^{1}\left(B_{R}\right)^{2}}} \\
& =\|g\|_{H^{-1 / 2}\left(\Gamma_{R}\right)^{2}}+\|f\|_{L^{2}\left(B_{R}\right)^{2}},
\end{aligned}
$$

which completes the proof.

Definition 2.5. A bounded sesquilinear form $a(\cdot, \cdot)$ on some Hilbert space $X$ is called strongly elliptic if there exists a compact form $q(\cdot, \cdot)$ such that

$$
|\operatorname{Re} a(u, u)| \geq C|| u \|_{X}^{2}-q(u, u) \quad \text { for all } u \in X \text {. }
$$

2.2. Near-field scattering map. For given perturbed parameters $q \in \mathcal{K}$, we define the scattering operator $S: \mathcal{K} \rightarrow H^{1}\left(B_{R}\right)^{2}$ by $S(q)=u$, where $u \in H^{1}\left(B_{R}\right)^{2}$ is the unique weak solution of (2.7)-(2.8). It is easily seen that the map $S$ is nonlinear with respect to $q$. A direct application of Theorem 2.4 gives the following result.

Copyright $@$ by SIAM. Unauthorized reproduction of this article is prohibited. 
Lemma 2.6. The map $S$ is bounded with the estimate

$$
\|S(q)\|_{H^{1}\left(B_{R}\right)^{2}} \leq \gamma_{q}^{-1}\|g\|_{\left(H^{-1 / 2}\left(\Gamma_{R}\right)\right)^{2}},
$$

where $c>0$ is a constant.

Lemma 2.7. Given the perturbed parameters $q_{l} \in \mathcal{K}, l=1,2$, we have the estimate

$$
\left\|S\left(q_{1}\right)-S\left(q_{2}\right)\right\|_{H^{1}\left(B_{R}\right)^{2}} \leq c \gamma_{q_{1}}^{-1}\left\|q_{1}-q_{2}\right\|_{L^{\infty}\left(B_{R}\right)^{3}}\left\|u_{2}\right\|_{\left(H^{1}\left(B_{R}\right)\right)^{2}},
$$

where $u_{2}$ is the unique weak solution of the problem (2.7)-(2.8) with perturbed parameters $q_{2}$ and $c>0$ is a constant.

Proof. Let $u_{1}, u_{2} \in H^{1}\left(B_{R}\right)^{2}$ be the unique weak solutions of the problem (2.7)(2.8) with perturbed parameters $q_{1}$ and $q_{2}$, respectively. Set $w=u_{2}-u_{1}$. Then we have

$$
a_{q_{1}}(w, v)-\int_{\Gamma_{R}} \mathcal{B} w \cdot \bar{v} d s=-a_{q_{2}-q_{1}-1}\left(u_{2}, v\right) .
$$

Then the inf-sup condition (2.15) together with the boundedness of $a_{q_{2}-q_{1}-1}$ implies the desired estimate.

For any $\delta q:=\left(\delta q_{\lambda}, \delta q_{\mu}, \delta q_{\rho}\right)^{\top} \in \mathcal{K}$, assume that $w \in H^{1}\left(B_{R}\right)^{2}$ is the unique weak solution of the following variational problem:

$$
a_{q}(w, v)-\int_{\Gamma_{R}} \mathcal{B} w \cdot \bar{v} d s=-a_{\delta q-1}(u, v) \quad \text { for all } \quad v \in H^{1}\left(B_{R}\right)^{2} .
$$

Let the map $\mathcal{T}_{q}: \mathcal{K} \rightarrow H^{1}\left(B_{R}\right)^{2}$ be such that

$$
\mathcal{T}_{q}(\delta q)=w .
$$

Lemma 2.8. Given the perturbed parameters $q, \delta q \in \mathcal{K}$, we have the estimate

$$
\left\|S(q+\delta q)-S(q)-\mathcal{T}_{q}(\delta q)\right\|_{H^{1}\left(B_{R}\right)^{2}} \leq C\|\delta q\|_{L^{\infty}\left(B_{R}\right)^{3}}^{2}\|g\|_{H^{-1 / 2}\left(\Gamma_{R}\right)^{2}},
$$

where $C>0$ is a constant.

Proof. Let $u_{1}, u_{2} \in H^{1}\left(B_{R}\right)^{2}$ be the unique weak solutions of the problem (2.7)(2.8) with perturbed parameters $q$ and $q+\delta q$, respectively. Let $\widetilde{u}=u_{2}-u_{1}$; it follows that

$$
a_{q}(\widetilde{u}-w, v)-\int_{\Gamma_{R}} \mathcal{B}(\widetilde{u}-w) \cdot \bar{v} d s=-a_{\delta q-1}(\widetilde{u}, v) \quad \text { for all } \quad v \in\left(H^{1}\left(B_{R}\right)\right)^{2} .
$$

Then we have the estimate

$$
\begin{aligned}
\|\widetilde{u}-w\|_{H^{1}\left(B_{R}\right)^{2}} & \leq c\|\delta q\|_{L^{\infty}\left(B_{R}\right)^{3}}\|\widetilde{u}\|_{\left(H^{1}\left(B_{R}\right)\right)^{2}} \\
& \leq c \gamma_{q}^{-1}\|\delta q\|_{L^{\infty}\left(B_{R}\right)^{3}}^{2}\left\|u_{2}\right\|_{\left(H^{1}\left(B_{R}\right)\right)^{2}} \\
& \leq c \gamma_{q}^{-1} \gamma_{q+\delta_{q}}^{-1}\|\delta q\|_{L^{\infty}\left(B_{R}\right)^{3}}^{2}\|g\|_{\left(H^{-1 / 2}\left(\Gamma_{R}\right)\right)^{2}} \\
& \leq C\|\delta q\|_{L^{\infty}\left(B_{R}\right)^{3}}^{2}\|g\|_{\left(H^{-1 / 2}\left(\Gamma_{R}\right)\right)^{2}},
\end{aligned}
$$

where $C>0$ is a constant defined as

$$
C:=c c_{0}^{2}, \quad c_{0}:=\sup _{q, \delta q \in \mathcal{K}}\left\{\gamma_{q}^{-1}, \gamma_{q+\delta q}^{-1}\right\}<\infty .
$$

Copyright $@$ ㅇ by SIAM. Unauthorized reproduction of this article is prohibited. 
Let $\gamma: H^{1}\left(B_{R}\right)^{2} \rightarrow H^{1 / 2}\left(\Gamma_{R}\right)^{2}$ be the trace operator to the boundary $\Gamma_{R}$ and define the near-field scattering map $N$ as $N(q)=\gamma S(q)$. By combining Lemmas $2.6-2.8$, we arrive at the following theorem.

THEOREM 2.9. The near-field scattering map $N$ is Fréchet differentiable with respect to $q$, and its Fréchet derivative is $N_{q}^{\prime}=\gamma \mathcal{T}_{q}$.

3. Inverse medium problem. In this section, we consider the inverse medium scattering problem of reconstructing the unknown perturbed elastic parameters and develop a Landweber iterative method. Assume that the total-field data $u$ is available over a range of frequencies $\omega \in\left[\omega_{\min }, \omega_{\max }\right]$ which can be divided into $\omega_{\min }=\omega_{1}<$ $\omega_{2}<\cdots<\omega_{N-1}<\omega_{N}=\omega_{\max }$ and over a range of incident directions $\theta^{\text {in }} \in$ $\left[\theta_{\min }, \theta_{\max }\right]$ which can be divided into $\theta_{\min }=\theta_{1}<\theta_{2}<\cdots<\theta_{M-1}<\theta_{M}=\theta_{\max }$. Let $u^{i, j}:=\left.u\left(x, \omega_{i}, \theta_{j}\right)\right|_{\Gamma_{R}}$ be the unique solution of the direct scattering problem with $\omega=\omega_{i}$ and $\theta^{i n}=\theta_{j}$. Consider the following inverse medium problem.

(IP) Given the elastic parameters $\lambda_{0}, \mu_{0}$, and $\rho_{0}$ of the background medium, reconstruct the perturbed Lamé parameters $q_{\lambda}, q_{\mu}$ and perturbed density $q_{\rho}$ from the multifrequency measurements $u^{i, j}, i=1, \ldots, N, j=1, l \cdots, M$.

The inverse problem (IP) can be formulated as follows: Given $\lambda_{0}, \mu_{0}$, and $\rho_{0}$, find $q_{\lambda}, q_{\mu}$, and $q_{\rho}$ such that

$$
N(q)=u^{i, j}, q=\left\{q_{\lambda}, q_{\mu}, q_{\rho}\right\} \quad \text { for } \quad i=1, \ldots, N, j=1, \ldots, M .
$$

In particular, the nonlinearity and ill-posedness of the inverse problem cause mathematical challenges from both theoretical and computational points of view. The nonlinearity leads to a nonconvex optimization problem, and the ill-posedness requires a certain form of regularization to get a reasonable approximation. Here, to solve the operator equation (3.1), we apply the Landweber iteration method taking the form [23]

$$
q_{k+1}=q_{k}+\alpha\left(N_{q_{k}}^{\prime}\right)^{*}\left(N(q)-N\left(q_{k}\right)\right), \quad k=0,1,2, \ldots,
$$

where $\alpha$ is the step size parameter.

3.1. Multifrequency iterative algorithm. For the adjoint of the operator $N_{q}^{\prime}$, we have the following result.

THEOREM 3.1. Let $u \in H^{1}\left(B_{R}\right)^{2}$ be the unique weak solution of (2.7)-(2.8). Then for any $h \in H^{-1 / 2}\left(\Gamma_{R}\right)^{2}$,

$$
\left(N_{q}^{\prime}\right)^{*}(h)=\left\{-\lambda_{0}(\nabla \cdot \bar{u})(\nabla \cdot \varphi),-2 \mu_{0} \mathcal{E}(\bar{u}): \mathcal{E}(\varphi), \rho_{0} \omega^{2} \bar{u} \cdot \varphi\right\},
$$

where $\varphi \in H^{1}\left(B_{R}\right)^{2}$ is the unique weak solution of the following boundary value problem:

$$
\begin{aligned}
& \nabla \cdot \sigma_{q_{\lambda}, q_{\mu}}(\bar{\varphi})+\rho_{0}\left(1+q_{\rho}\right) \omega^{2} \bar{\varphi}=0 \text { in } \quad B_{R} \\
& T_{\lambda_{0}, \mu_{0}} \bar{\varphi}-\mathcal{B} \bar{\varphi}=\bar{h} \quad \text { on } \quad \Gamma_{R} .
\end{aligned}
$$

Proof. For any $\delta q \in \mathcal{K}$, let $w \in\left(H^{1}\left(B_{R}\right)\right)^{2}$ be the unique weak solution of the following variational problem:

$$
a_{q}(w, v)-\int_{\Gamma_{R}} \mathcal{B} w \cdot \bar{v} d s=-a_{\delta q-1}(u, v) \quad \text { for all } \quad v \in\left(H^{1}\left(B_{R}\right)\right)^{2} .
$$

Copyright (C) by SIAM. Unauthorized reproduction of this article is prohibited. 
Replacing $v$ by $\varphi$, we obtain

$$
\begin{aligned}
& A_{\delta q_{\lambda}-1}(u, \varphi)+B_{\delta q_{\mu}-1}(u, \varphi)-\int_{B_{R}} \rho_{0} \delta q_{\rho} \omega^{2} u \cdot \bar{\varphi} d x \\
= & -A_{q_{\lambda}}(w, \varphi)-B_{q_{\mu}}(w, \varphi)+\int_{B_{R}} \rho_{0}\left(1+q_{\rho}\right) \omega^{2} w \cdot \bar{\varphi} d x+\langle\mathcal{B} w, \varphi\rangle_{-1 / 2,1 / 2} \\
= & -A_{q_{\lambda}}(w, \varphi)-B_{q_{\mu}}(w, \varphi)+\int_{B_{R}} \rho_{0}\left(1+q_{\rho}\right) \omega^{2} w \cdot \bar{\varphi} d x+\left\langle w, \mathcal{B}^{*} \varphi\right\rangle_{1 / 2,-1 / 2} \\
= & -A_{q_{\lambda}}(w, \varphi)-B_{q_{\mu}}(w, \varphi)+\int_{B_{R}} \rho_{0}\left(1+q_{\rho}\right) \omega^{2} w \cdot \bar{\varphi} d x+\langle w, \overline{\mathcal{B}} \bar{\varphi}\rangle_{1 / 2,-1 / 2} \\
= & -\langle w, h\rangle_{1 / 2,-1 / 2} \\
= & \left.-\left\langle N_{q}^{\prime}(\delta q), h\right)\right\rangle_{1 / 2,-1 / 2} \\
= & -\int_{B_{R}} \delta q \cdot \overline{\left(N_{q}^{\prime}\right)^{*} h} d x
\end{aligned}
$$

in which we have used the relation $(2.6)$ and $\langle\cdot, \cdot\rangle_{s,-s}$ denotes the $L^{2}$ duality pairing between $H^{s}(\Gamma)^{2}$ and $H^{-s}(\Gamma)^{2}$. Since it holds for any $\delta q \in \mathcal{K}$, we complete the proof. $\square$

Denote

$$
q_{i, j, l}=\left\{q_{\lambda}^{i, j, l}, q_{\mu}^{i, j, l}, q_{\rho}^{i, j, l}\right\},
$$

where the indices $i, j, l$ are related to the frequency $\omega_{i}$, the incident direction $\theta_{j}$, and the current inner Landweber iteration number. Given initial guesses $q_{1,1,0}=0$, we now describe a procedure that determines a better approximation $q_{i, j}:=q_{i, j, L}$ at the frequency $\omega=\omega_{i}$ with incident direction $\theta^{i n}=\theta_{j}$ for $i=1, \ldots, N, j=1, \ldots, M$ in an increasing manner. For each $i, j$, we apply $L$ steps of Landweber iterations, i.e., $l=1, \ldots, L$. For fixed $i, j$, suppose now that an approximation of the scatterer $q_{i, j, l-1}$ has been recovered. For the recovered scatterer $q_{i, j, l-1}$, we solve at $\omega=\omega_{i}$ and $\theta^{i n}=\theta_{j}$ the direct problem

$$
\begin{aligned}
\nabla \cdot \sigma_{q_{\lambda}^{i, j, l-1}, q_{\mu}^{i, j, l-1}}\left(\widetilde{u}^{i, j, l}\right)+\rho_{0}\left(1+q_{\rho}^{i, j, l-1}\right) \omega_{i}^{2} \widetilde{u}^{i, j, l}=0 & \text { in } \quad B_{R}, \\
T_{\lambda_{0}, \mu_{0}} \widetilde{u}^{i, j, l}-\mathcal{B} \widetilde{u}^{i, j, l}-g=0 & \text { on } \Gamma_{R} .
\end{aligned}
$$

Then for any $h \in H^{1 / 2}\left(\Gamma_{R}\right)^{2}$,

$$
\begin{aligned}
& \left(N_{q_{i, j, l-1}}^{\prime}\right)^{*}(h) \\
= & \left\{-\lambda_{0}\left(\nabla \cdot \overline{\widetilde{u}^{i, j, l}}\right)\left(\nabla \cdot \varphi^{i, j, l}\right),-2 \mu_{0} \mathcal{E}\left(\overline{\widetilde{u}^{i, j, l}}\right): \mathcal{E}\left(\varphi^{i, j, l}\right), \rho_{0} \omega_{i}^{2} \overline{\widetilde{u}^{i, j, l}} \cdot \varphi^{i, j, l}\right\},
\end{aligned}
$$

where $\varphi^{i, j, l} \in H^{1}\left(B_{R}\right)^{2}$ is the unique weak solution of the following boundary value problem:

$$
\begin{aligned}
& \nabla \cdot \sigma_{q_{\lambda}^{i, j, l-1}, q_{\mu}^{i, j, l-1}}\left(\overline{\varphi^{i, j, l}}\right)+\rho_{0}\left(1+q_{\rho}^{i, j, l-1}\right) \omega_{i}^{2} \overline{\varphi^{i, j, l}}=0 \text { in } \quad B_{R}, \\
& T_{\lambda_{0}, \mu_{0}} \overline{\varphi^{i, j, l}}-\mathcal{B} \overline{\varphi^{i, j, l}}=\bar{h} \quad \text { on } \quad \Gamma_{R} .
\end{aligned}
$$

Then the Landweber iteration leads to

$$
q_{i, j, l}=q_{i, j, l-1}+\alpha\left(N_{q_{i, j, l-1}}^{\prime}\right)^{*}\left(u^{i, j}-\widetilde{u}^{i, j, l}\right), \quad l=1, \ldots, N .
$$

Copyright $@$ by SIAM. Unauthorized reproduction of this article is prohibited. 
Note that the elastic parameters are all real values. Therefore, at each step of iteration, we apply a simple regularization as

$$
q_{i, j, l} \leftarrow \operatorname{Re}\left\{q_{i, j, l}\right\} .
$$

The multifrequency iterative algorithm for solving the inverse medium scattering is summarized in Algorithm 1.

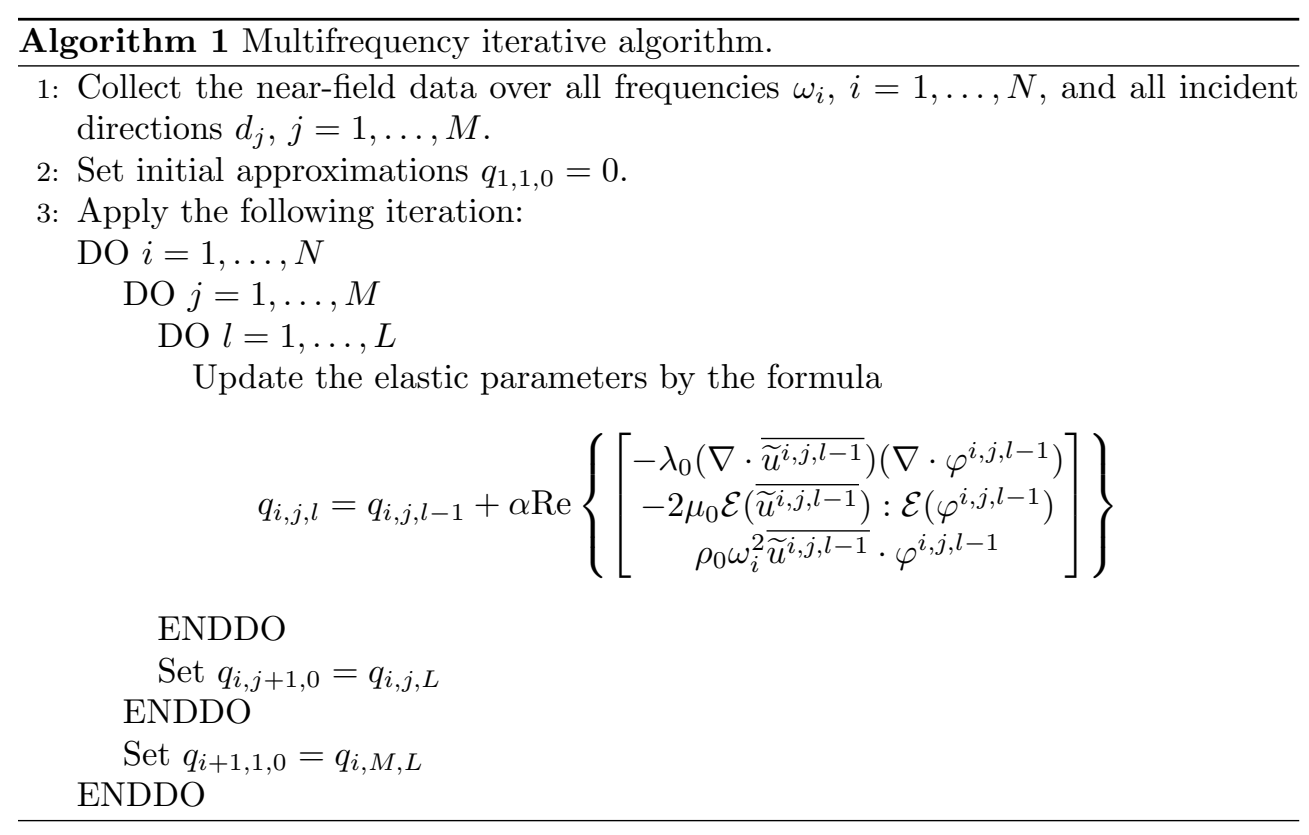

3.2. Multifrequency iterative algorithm from phaseless data. We now consider the reconstruction from phaseless data. Define the phaseless near-field scattering map $F: \mathcal{K} \rightarrow L^{2}\left(\Gamma_{R}\right)^{2}$ by

$$
F(q)=\left.|u|_{\Gamma_{R}}\right|^{2}=\overline{N(q)} \cdot N(q) .
$$

Lemмa 3.2. The phaseless near-field scattering map $F$ is Fréchet differentiable with respect to $q$, and its Fréchet derivative is given by

$$
F_{q}^{\prime}(\cdot)=2 \operatorname{Re}\left\{\overline{N(q)} \cdot N_{q}^{\prime}(\cdot)\right\} .
$$

The adjoint of $F_{q}^{\prime}$ is given in the following theorem.

TheOREM 3.3. Let $u \in H^{1}\left(B_{R}\right)^{2}$ be the unique weak solution of (2.7)-(2.8). Then for any $\hbar \in L^{2}\left(\Gamma_{R}\right)^{2}$,

$$
\left(F_{q}^{\prime}\right)^{*}(\hbar)=2 \operatorname{Re}\left\{-\lambda_{0}(\nabla \cdot \bar{u})(\nabla \cdot \psi),-2 \mu_{0} \mathcal{E}(\bar{u}): \mathcal{E}(\psi), \rho_{0} \omega^{2} \bar{u} \cdot \psi\right\},
$$

where $\psi \in H^{1}\left(B_{R}\right)^{2}$ is the unique weak solution of the following boundary value problem:

$$
\begin{aligned}
\nabla \cdot \sigma_{q_{\lambda}, q_{\mu}}(\bar{\varphi})+\rho_{0}\left(1+q_{\rho}\right) \omega^{2} \bar{\varphi} & =0 \quad \text { in } \quad B_{R}, \\
T_{\lambda_{0}, \mu_{0}} \bar{\varphi}-\mathcal{B} \bar{\varphi} & =\overline{\hbar u} \quad \text { on } \quad \Gamma_{R} .
\end{aligned}
$$

Copyright (c) by SIAM. Unauthorized reproduction of this article is prohibited. 
Proof. The proof is similar to that of Theorem 3.1, and so we omit it here.

We now describe the Landweber iterative algorithm based on phaseless data. Use the same notations as in section 3.1. For any $\hbar \in H^{1 / 2}\left(\Gamma_{R}\right)$, let $\psi^{i, j, l} \in H^{1}\left(B_{R}\right)^{2}$ be the unique weak solution of the following boundary value problem:

$$
\begin{aligned}
& \nabla \cdot \sigma_{q_{\lambda}^{i, j, l-1}, q_{\mu}^{i, j, l-1}}\left(\overline{\psi^{i, j, l}}\right)+\rho_{0}\left(1+q_{\rho}^{i, j, l-1}\right) \omega_{i}^{2} \overline{\psi^{i, j, l}}=0 \quad \text { in } \quad B_{R}, \\
& T_{\lambda_{0}, \mu_{0}} \overline{\psi^{i, j, l}}-\mathcal{B} \overline{\psi^{i, j, l}}=\overline{\hbar \widetilde{u}^{i, j, l}} \quad \text { on } \quad \Gamma_{R} \text {. }
\end{aligned}
$$

Then the Landweber iteration leads to

$$
q_{i, j, l}=q_{i, j, l-1}+\alpha\left(F_{q_{i, j, l-1}}^{\prime}\right)^{*}\left(\left|u^{i, j}\right|^{2}-\left.\left|\widetilde{u}^{i, j, l}\right|_{\Gamma_{R}}\right|^{2}\right), \quad l=1, \ldots, N .
$$

The algorithm for solving the inverse medium scattering from phaseless data is summarized in Algorithm 2.

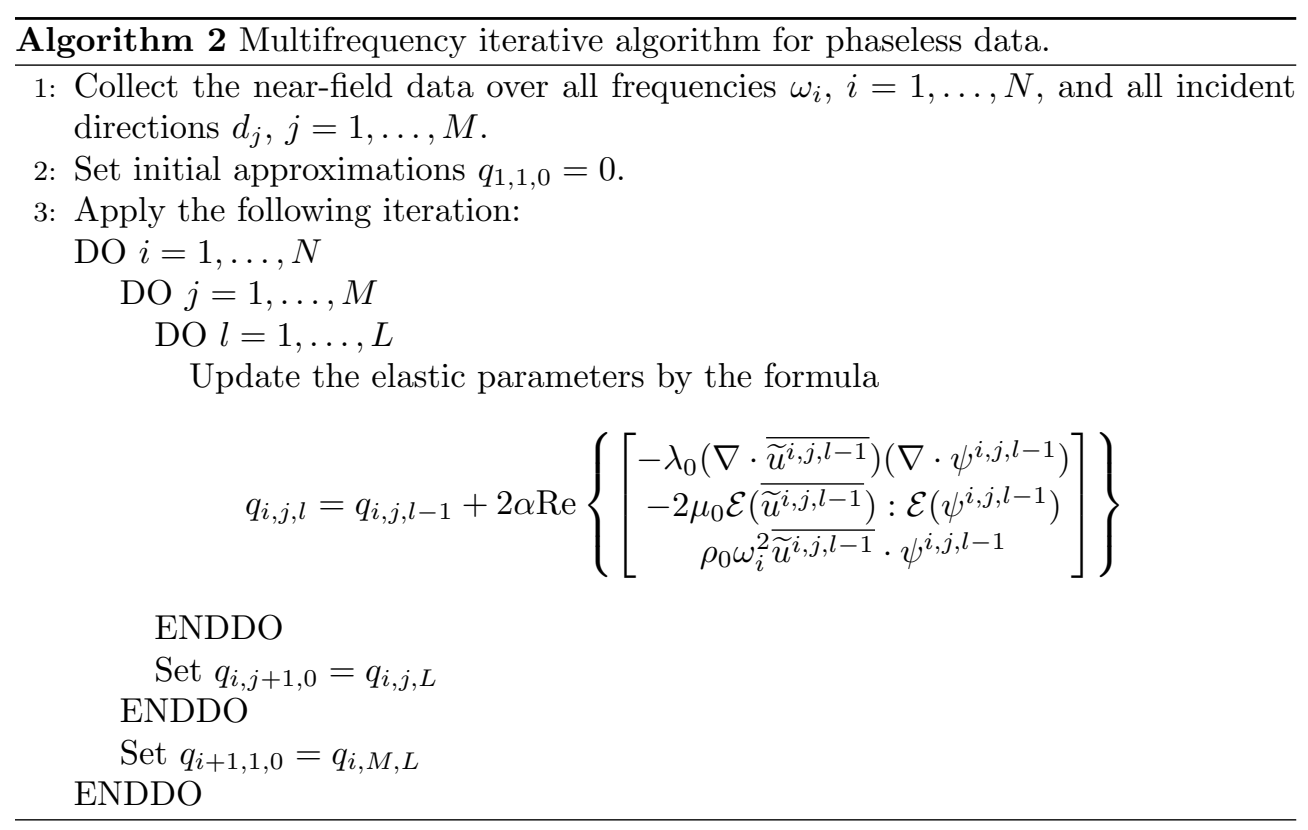

3.3. Convergence. In this section, we briefly discuss the convergence of the proposed algorithms based on the classical analysis of the Landweber iteration method for nonlinear ill-posed problems. For more detailed analyses of the Landweber iteration and its modification form, we refer the reader to [23, 29] and the references therein. It should be pointed out that although we apply the multifrequency strategy in this paper, it is extremely difficult to investigate the dependence of the convergence of the algorithms on the number and interval of the selected frequencies. For the corresponding analysis of the inverse medium scattering problems in acoustics that takes a similar form as the special case discussed in section 4, we refer the reader to [16].

Due to the difficulty of investigating the phaseless case, we only discuss the phased case here. Consider the following operator equation (containing the dependence on frequency):

$$
N(q, \omega)=y(\omega), \quad N: \mathcal{D}(N) \times\left[\omega_{\min }, \omega_{\max }\right] \rightarrow Y,
$$

Copyright $@$ by SIAM. Unauthorized reproduction of this article is prohibited. 
where $\mathcal{D}(N) \subset X$ and $X, Y$ are all Hilbert spaces with inner products $\langle\cdot, \cdot\rangle_{X},\langle\cdot, \cdot\rangle_{Y}$ and norms $\|\cdot\|_{X},\|\cdot\|_{Y}$, respectively. In particular, $X=L^{2}\left(B_{R}\right)^{3}, Y=H^{1 / 2}\left(\Gamma_{R}\right)^{2}$, and $y(\omega)=\left.u\right|_{\Gamma_{R}}$. As mentioned above, the operator equation (3.6) is strictly nonlinear and ill-posed. Then the nonlinear Landweber iteration takes the form

$$
q_{k+1}=q_{k}+\alpha\left(N_{q_{k}}^{\prime}\right)^{*}\left(y-N\left(q_{k}\right)\right), \quad k=0,1,2, \ldots,
$$

for exact data $y$ and the form

$$
q_{k+1}^{\delta}=q_{k}^{\delta}+\alpha\left(N_{q_{k}^{\delta}}^{\prime}\right)^{*}\left(y^{\delta}-N\left(q_{k}^{\delta}\right)\right), \quad k=0,1,2, \ldots,
$$

for inexact data $y^{\delta}$ satisfying $\sup _{\omega \in\left[\omega_{\min }, \omega_{\max }\right]}\left\{\left\|y^{\delta}-y\right\|_{Y}\right\} \leq \delta$.

Let $\widetilde{B}_{r}\left(q_{0}\right)$ denote a closed ball of radius $r$ around $q_{0}$. Assume that $\alpha>0$ is sufficiently small such that $\alpha\left\|N_{q}^{\prime}\right\|<1$ in $\widetilde{B}_{2 r}\left(q_{0}\right)$. For simplicity, we consider $\alpha=1$ and $\left\|N_{q}^{\prime}\right\|<1$. Resulting from the regularity theory, we can obtain that $u \in H_{\text {loc }}^{2}\left(\mathbb{R}^{2} \backslash \overline{B_{R-\epsilon}}\right)^{2}$ for any small $\epsilon>0$ such that $\sup \{q\} \subset B_{R-\epsilon}$, which implies that $u \in H^{3 / 2}\left(\Gamma_{R}\right)^{2} \hookrightarrow H^{1 / 2}\left(\Gamma_{R}\right)^{2}$. Thus, the operator $F$ here is compact. According to the analysis in Lemma 2.8 and the trace theorem, letting $r>0$ and $q, \widetilde{q} \in \widetilde{B}_{2 r}\left(q_{0}\right) \subset$ $\mathcal{D}(N)$ satisfying $\|q-\widetilde{q}\|_{L^{\infty}\left(B_{R}\right)^{3}} \leq \epsilon$ with sufficiently small $\epsilon>0$, it holds that

$$
\left\|N(\widetilde{q})-N(q)-N_{q}^{\prime}(\widetilde{q}-q)\right\|_{Y} \leq \eta\|N(\widetilde{q})-N(q)\|_{Y}, \quad 0<\eta<c_{0}<\frac{1}{2} .
$$

In fact, from the proof of Lemma 2.8 and the boundedness of trace operator $\gamma$, we can obtain that

$$
\begin{aligned}
& \left\|N(\widetilde{q})-N(q)-N_{q}^{\prime}(\widetilde{q}-q)\right\|_{H^{1 / 2}\left(\Gamma_{R}\right)^{2}} \\
\leq & \|\gamma\|\left\|S(\widetilde{q})-S(q)-\mathcal{T}_{q}(\widetilde{q}-q)\right\|_{H^{1}\left(B_{R}\right)^{2}} \\
\leq & C\|\delta q\|_{L^{\infty}\left(B_{R}\right)^{3}}\|S(\widetilde{q})-S(q)\|_{H^{1}\left(B_{R}\right)^{2}} \\
\leq & C \epsilon\|N(\widetilde{q})-N(q)\|_{H^{1 / 2}\left(\Gamma_{R}\right)^{2}} .
\end{aligned}
$$

The convergence of the Landweber iteration is given in the following theorem; see Corollary 2.3 and Theorem 2.4 in [29].

Theorem 3.4. Assume that $N(q)=y$ is solvable in $\widetilde{B}_{r}\left(q_{0}\right)$. Then the nonlinear Landweber iteration (3.7) converges to a solution of $N(q)=y$. Furthermore, let $q^{\dagger}$ be such that $\mathcal{N}\left(N_{q^{\dagger}}^{\prime}\right) \subset \mathcal{N}\left(N_{q}^{\prime}\right)$ for all $q \in \widetilde{B}_{r}\left(q^{\dagger}\right)$; then $q_{k}$ converges to $q^{\dagger}$ as $k \rightarrow+\infty$. Here, $\mathcal{N}(\cdot)$ denotes the nullspace.

Remark 3.1. It follows immediately from (3.9) that

$$
\frac{1}{1+\eta}\left\|N_{q}^{\prime}(\widetilde{q}-q)\right\|_{Y} \leq\|N(\widetilde{q})-N(q)\|_{Y} \leq \frac{1}{1-\eta}\left\|N_{q}^{\prime}(\widetilde{q}-q)\right\|_{Y}
$$

This further implies the existence of a unique solution of minimal distance to $q_{0}$ if $N(q)=y$ is solvable in $\widetilde{B}_{r}\left(q_{0}\right)$.

In the case of inexact data, the iteration procedure should be combined with a stopping rule in order to act as a regularization method. For example, for each frequency, one can employ the discrepancy principle; i.e., the iteration is stopped after $k^{\prime}$ steps with

$$
\left\|y^{\delta}-N\left(q_{k^{\prime}}^{\delta}\right)\right\|_{Y} \leq \tau \delta<\left\|y^{\delta}-N\left(q_{k}^{\delta}\right)\right\|_{Y}
$$

Copyright $@$ by SIAM. Unauthorized reproduction of this article is prohibited. 
where $\tau$ is an appropriately chosen positive number. Before stating the convergence of the iteration (3.8) for the inexact data case, we need to discuss the selection of $\tau$. Note that the parameter $\eta$ must be dependent on $\omega$.

Proposition 3.5. Assume $N(q)=y$ has a solution $q_{*} \in \widetilde{B}_{r}\left(q_{0}\right)$ and $q_{k}^{\delta} \in \widetilde{B}_{r}\left(q_{*}\right)$.

(i) A sufficient condition for $q_{k+1}^{\delta}$ generated from (3.8) to be a better approximation of $q_{*}$ than $q_{k}^{\delta}$ is that

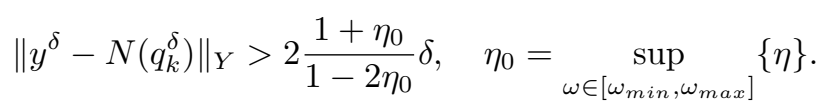

This further leads to the selection of $\tau$ as

$$
\tau>2 \frac{1+\eta_{0}}{1-2 \eta_{0}}
$$

(ii) At each frequency, let $k^{\prime}$ be chosen according to the stopping rule; we have

$$
k^{\prime}(\tau \delta)^{2}<\sum_{k=0}^{k^{\prime}-1}\left\|y^{\delta}-N\left(q_{k}^{\delta}\right)\right\|_{Y}^{2} \leq \frac{\tau}{\left(1-2 \eta_{0}\right) \tau-2\left(1+\eta_{0}\right)}\left\|q_{0}-q_{*}\right\|_{X}^{2} .
$$

Proof. The proof is similar to that of Proposition 2.2 and Corollary 2.3 in [29]. In fact, following the same steps of deriving (2.13) in [29], it holds that

$$
\begin{aligned}
& \left\|q_{k+1}^{\delta}-q_{*}\right\|_{X}^{2}-\left\|q_{k}^{\delta}-q_{*}\right\|_{X}^{2} \\
= & 2\left\langle q_{k+1}^{\delta}-q_{k}^{\delta}, q_{k}^{\delta}-q_{*}\right\rangle_{X}+\left\|q_{k+1}^{\delta}-q_{k}^{\delta}\right\|_{X}^{2} \\
= & 2\left\langle y^{\delta}-N\left(q_{k}^{\delta}\right), N_{q_{k}^{\delta}}^{\prime}\left(q_{k}^{\delta}-q_{*}\right)\right\rangle_{Y}+\left\|\left(N_{q_{k}^{\delta}}^{\prime}\right)^{*}\left(y^{\delta}-N\left(q_{k}^{\delta}\right)\right)\right\|_{X}^{2} \\
\leq & 2\left\langle y^{\delta}-N\left(q_{k}^{\delta}\right), y^{\delta}-N\left(q_{k}^{\delta}\right)-N_{q_{k}^{\delta}}^{\prime}\left(q_{*}-q_{k}^{\delta}\right)\right\rangle_{Y}-\left\|y^{\delta}-N\left(q_{k}^{\delta}\right)\right\|_{Y}^{2} \\
\leq & \left\|y^{\delta}-N\left(q_{k}^{\delta}\right)\right\|_{Y}\left(2 \delta+2 \eta\left\|y-N\left(q_{k}^{\delta}\right)\right\|_{Y}-\left\|y^{\delta}-N\left(q_{k}^{\delta}\right)\right\|_{Y}\right) \\
\leq & \left\|y^{\delta}-N\left(q_{k}^{\delta}\right)\right\|_{Y}\left(2 \delta+2 \eta_{0}\left\|y-N\left(q_{k}^{\delta}\right)\right\|_{Y}-\left\|y^{\delta}-N\left(q_{k}^{\delta}\right)\right\|_{Y}\right) \\
\leq & \left\|y^{\delta}-N\left(q_{k}^{\delta}\right)\right\|_{Y}\left[2\left(1+\eta_{0}\right) \delta-\left(1-2 \eta_{0}\right)\left\|y^{\delta}-N\left(q_{k}^{\delta}\right)\right\|_{Y}\right],
\end{aligned}
$$

which completes the proof of (i) under the sufficient condition. Furthermore, the special choice of $\tau$ implies that

$$
\begin{aligned}
& \left\|q_{k+1}^{\delta}-q_{*}\right\|_{X}-\left\|q_{k}^{\delta}-q_{*}\right\|_{X} \\
\leq & {\left[2 \tau^{-1}\left(1+\eta_{0}\right)+2 \eta_{0}-1\right]\left\|y^{\delta}-N\left(q_{k}^{\delta}\right)\right\|_{Y}^{2} . }
\end{aligned}
$$

Adding up these inequalities for $k$ from 0 through $k^{\prime}-1$ leads to

$$
\begin{aligned}
& {\left[1-2 \eta_{0}-2 \tau^{-1}\left(1+\eta_{0}\right)\right] \sum_{k=0}^{k^{\prime}-1}\left\|y^{\delta}-N\left(q_{k}^{\delta}\right)\right\|_{Y}^{2} } \\
\leq & \left\|q_{0}-q_{*}\right\|_{X}^{2}-\left\|q_{k^{\prime}}^{\delta}-q_{*}\right\|_{X}^{2} .
\end{aligned}
$$

Then (ii) can be proved by combining this inequality and the stopping rule.

Now we can have the following convergence result for inexact data; see Theorem 2.6 in $[29]$.

Copyright (c) by SIAM. Unauthorized reproduction of this article is prohibited. 
Theorem 3.6. Assume that $N(q)=y$ is solvable in $\widetilde{B}_{r}\left(q_{0}\right)$ and let $k^{\prime}$ be chosen according to the stopping rule. The nonlinear Landweber iteration (3.8) converges to a solution of $N(q)=y$. Let $q^{\dagger}$ be such that $\mathcal{N}\left(N_{q^{\dagger}}^{\prime}\right) \subset \mathcal{N}\left(N_{q}^{\prime}\right)$ for all $q \in \widetilde{B}_{r}\left(q^{\dagger}\right)$; then $q_{k^{\prime}}^{\delta}$ converges to $q^{\dagger}$ as $\delta \rightarrow 0$.

Remark 3.2. According to the convergence of the Landweber iteration, the choice of the initial approximation may affect the efficiency of the algorithms. In fact, in this paper we reconstruct the relative factors of the elastic parameters of the inhomogeneous medium compared with the parameters of the background medium-not the elastic parameters of the inhomogeneous medium themselves; i.e., we reconstruct

$$
q_{\lambda}=\frac{\lambda}{\lambda_{0}}-1, \quad q_{\mu}=\frac{\mu}{\mu_{0}}-1, \quad q_{\rho}=\frac{\rho}{\rho_{0}}-1,
$$

and they are of $\mathcal{O}(1)$. Thus, a simple choice of the initial approximation, that we applied in this paper, is $q_{\lambda}=q_{\mu}=q_{\rho}=0$, which means that there is no inhomogeneity, and it can be seen from the numerical examples that this choice works well. As suggested in [12] for the inverse medium problems in acoustics, one can obtain an initial guess from the Born approximation, and the scattering data with the higher frequency must be used in order to recover more Fourier modes of the true scatterer.

4. Discussion of a special case. In this section, we briefly discuss the special case when $q_{\lambda}=q_{\mu}=0$. In this case, the considered inverse medium problem in elasticity is consistent with that in acoustics and electromagnetics discussed in $[7,8$, $9,10,11]$.

Alternatively, one can consider near-field scattering map $\widetilde{N}$ as $\widetilde{N}\left(q_{\rho}\right)=\left.\widetilde{u}\right|_{\Gamma_{R}}$, where $u=u^{s c}+u^{i n} \in H^{1}\left(B_{R}\right)^{2}$ is the unique weak solution of (2.7)-(2.8). Following the steps discussed in sections 2 and 3 (see also in [7]), we can obtain the following result.

THEOREM 4.1. The near-field scattering maps $N, \widetilde{N}$ are Fréchet differentiable with respect to $q_{\rho}$, and their Fréchet derivatives are given by

$$
N_{q_{\rho}}^{\prime}\left(\delta q_{\rho}\right)=\tilde{N}_{q_{\rho}}^{\prime}\left(\delta q_{\rho}\right)=\left.v\right|_{\Gamma_{R}},
$$

where $v \in H^{1}\left(B_{R}\right)^{2}$ is the unique weak solution of the following boundary value problem:

$$
\begin{aligned}
\Delta_{\lambda_{0}, \mu_{0}}^{*} v+\rho_{0} \omega^{2}\left(1+q_{\rho}\right) v & =-\rho_{0} \omega^{2} \delta q_{\rho}\left(u^{i n}+u^{s c}\right) \quad \text { in } \quad B_{R} \\
T_{\lambda_{0}, \mu_{0}} v & =\mathcal{B} v \quad \text { on } \quad \Gamma_{R} .
\end{aligned}
$$

Moreover, for any $h \in H^{1 / 2}\left(\Gamma_{R}\right)^{2}$,

$$
\left(N_{q_{\rho}}^{\prime}\right)^{*}(h)=\left(\tilde{N}_{q_{\rho}}^{\prime}\right)^{*}(h)=\rho_{0} \omega^{2} \overline{\left(u^{i n}+u^{s c}\right)} \cdot \varphi,
$$

where $\varphi \in H^{1}\left(B_{R}\right)^{2}$ is the unique weak solution of the following boundary value problem:

$$
\begin{aligned}
& \Delta_{\lambda_{0}, \mu_{0}}^{*} \bar{\varphi}+\rho_{0}\left(1+q_{\rho}\right) \omega^{2} \bar{\varphi}=0 \text { in } \quad B_{R}, \\
& T_{\lambda_{0}, \mu_{0}} \bar{\varphi}-\mathcal{B} \bar{\varphi}=\bar{h} \quad \text { on } \quad \Gamma_{R} .
\end{aligned}
$$

Similarly to Algorithm 1, we can summarize the multifrequency iterative algorithm for the reconstruction of inhomogeneous density function $q_{\rho}$ in Algorithm 3 . 


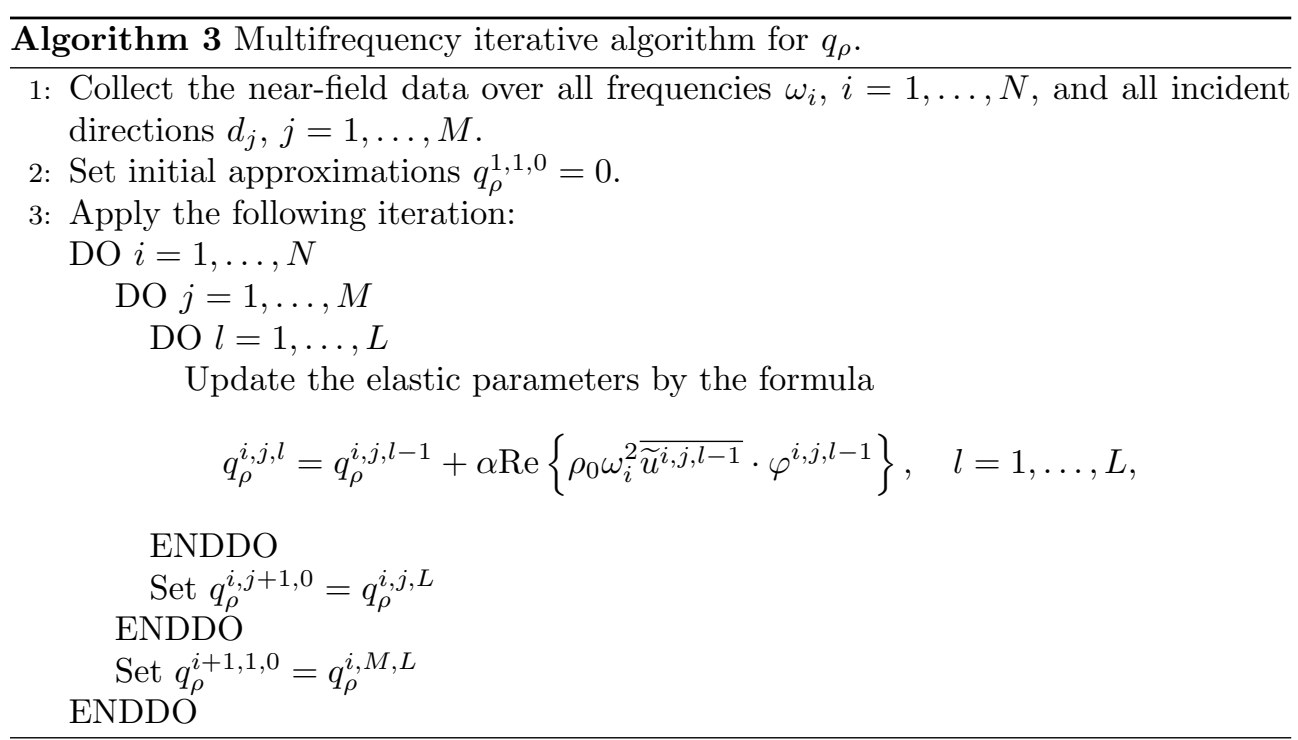

5. Numerical examples. In this section, we present several numerical examples to verify the accuracy and effectiveness of the recursive algorithm. We always choose $\lambda_{0}=2, \mu_{0}=1, \rho_{0}=1$, and $R=1$. The near-field measurements are obtained by using the finite element method to solve the forward scattering problem. Define the relative error

$$
e_{q}:=\frac{\|q-\widetilde{q}\|_{L^{2}\left(B_{R}\right)}}{\|q\|_{L^{2}\left(B_{R}\right)}},
$$

where $q$ and $\widetilde{q}$ are the true and reconstructed values of the parameter of the scatterer. The true values of the elastic parameters $q_{\lambda}, q_{\mu}, q_{\rho}$ are shown in Figure 1. Ten equally spaced frequencies are used in the construction, starting from the lowest frequency $\omega_{\min }=1$ and ending at the highest frequency $\omega_{\max }=10$. The number of incident directions is taken as $M=16$ and $\theta_{j}=2(j-1) \pi / M$ for $j=1, \ldots, M$. At each incident direction, 10 Landweber iteration steps are taken for one frequency. Corresponding to the stiffness tensor of the background elastic medium using Voigt notation, we choose the relaxation parameter $\alpha$ as a matrix

$$
\alpha=\frac{1}{100 \omega}\left[\begin{array}{ccc}
2+\lambda_{0} / \mu_{0} & \lambda_{0} / \mu_{0} & 0 \\
\lambda_{0} / \mu_{0} & 2+\lambda_{0} / \mu_{0} & 0 \\
0 & 0 & 1
\end{array}\right] .
$$

We collect the final reconstruction error for the following examples in Tables 1 and 2 . In addition, the number of iterations shown in the following figures ranges from 0 to $N \times M$.

Example 1. We consider the reconstruction from multifrequency measurements with multiple incident directions. The reconstructed elastic parameters are presented in Figure 2((a)-(c)) and Figure 3((a)-(c)). The relative errors shown in Figure 2((d)(f)) and Figure 3((d)-(f)) indicate that the relative errors decrease as frequency and number of iterations increase. However, it can be seen that if we choose plane shear incident waves, the reconstruction of $q_{\lambda}$, which can further affect the reconstructions of $q_{\mu}$ and $q_{\rho}$, is worse than that using plane pressure incident waves. A possible 
TABLE 1

Final reconstruction errors of $q_{\lambda}, q_{\mu}$, and $q_{\rho}$ for Examples $1-4$.

\begin{tabular}{|c|c|c|c|c|}
\hline Figure & Comment & Error of $q_{\lambda}$ & Error of $q_{\mu}$ & Error of $q_{\rho}$ \\
\hline 2 & Plane shear incidence & 0.74 & 0.36 & 0.39 \\
\hline 3 & Plane pressure incidence & 0.40 & 0.44 & 0.39 \\
\hline $4(\mathrm{a}, \mathrm{b}, \mathrm{c})$ & $3 \%$ noise & 0.43 & 0.47 & 0.42 \\
\hline $4(\mathrm{~d}, \mathrm{e}, \mathrm{f})$ & $5 \%$ noise & 0.44 & 0.47 & 0.42 \\
\hline 5 & Scale step size & 0.47 & 0.51 & 0.48 \\
\hline 6 & Phaseless measurement & 0.43 & 0.46 & 0.37 \\
\hline 7 & One $\theta^{\text {inc }}$ & 0.66 & 0.73 & 0.61 \\
\hline
\end{tabular}

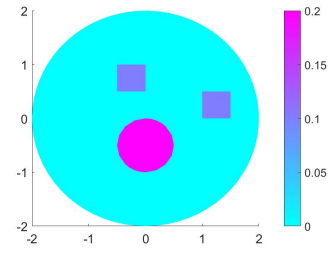

(a) $q_{\lambda}$

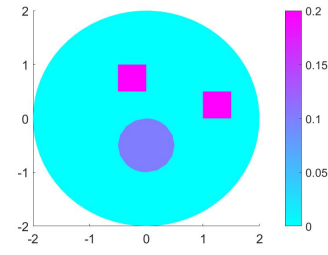

(b) $q_{\mu}$

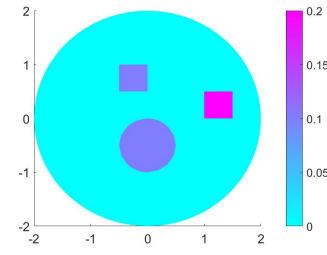

(c) $q_{\rho}$

FIG. 1. The exact value of perturbed parameters.

explanation for this phenomenon is that, in comparison with the plane pressure waves, the plane shear incident waves only contain the information of $\mu_{0}$ and $\rho_{0}$. In the following, we consider the plane pressure incident waves only. To verify the stability of our method, the reconstructions from noised data with noise levels $\delta=3 \%, 5 \%$ are presented in Figure 4.

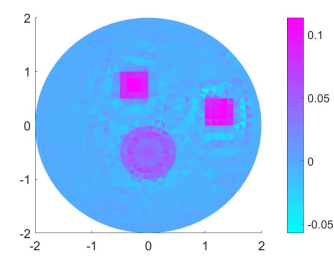

(a) $q_{\lambda}$

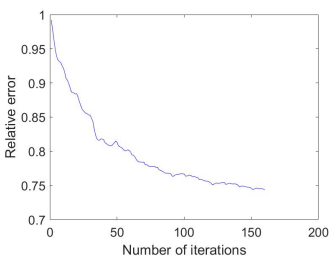

(d) $e_{q_{\lambda}}$

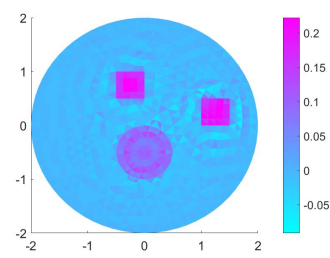

(b) $q_{\mu}$

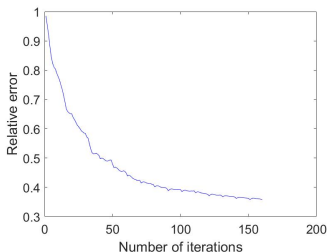

(e) $e_{q_{\mu}}$

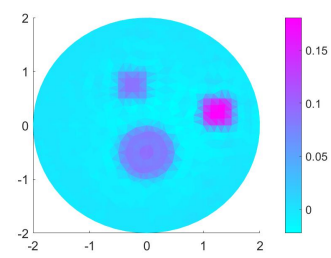

(c) $q_{\rho}$

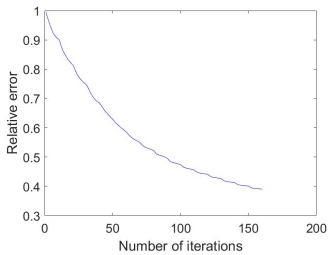

(f) $e_{q_{\rho}}$

FIG. 2. Example 1: The reconstruction of perturbed parameters with plane shear incident waves.

Example 2. Note that one can choose $\alpha$ as a scale value. For simplicity, we choose $\alpha=0.01 / \omega$, and the reconstruction results and the corresponding relative errors are presented in Figure 5. It can be seen that in this example, the iterations using the step size matrix (5.1) are more stable than those using scale step size. It should be pointed out that we cannot prove that, using the special choice of step size $\alpha$ which 


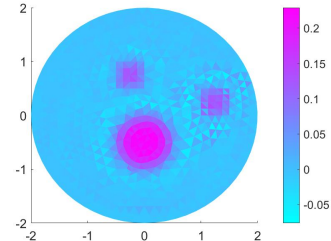

(a) $q_{\lambda}$

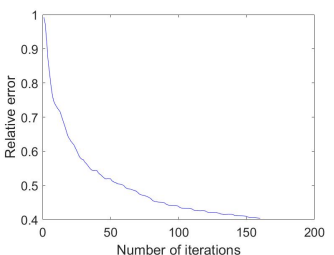

(d) $e_{q_{\lambda}}$

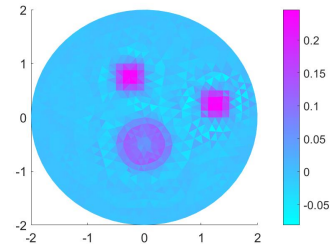

(b) $q_{\mu}$

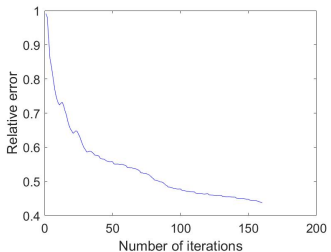

(e) $e_{q_{\mu}}$

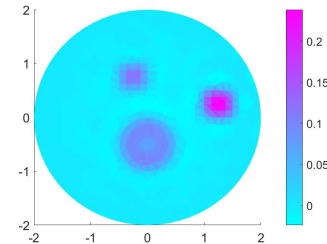

(c) $q_{\rho}$

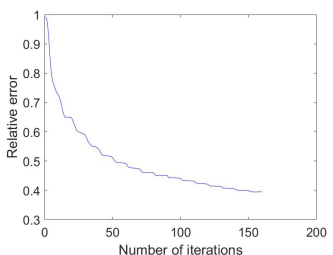

(f) $e_{q_{\rho}}$

FIG. 3. Example 1: The reconstruction of perturbed parameters with plane pressure incident waves.

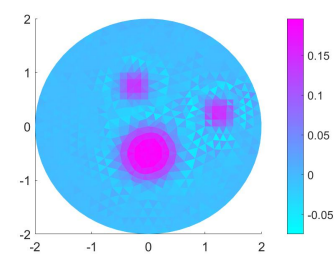

(a) $q_{\lambda}$

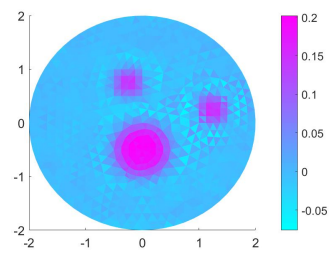

(d) $q_{\lambda}$

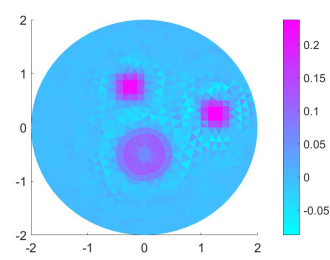

(b) $q_{\mu}$

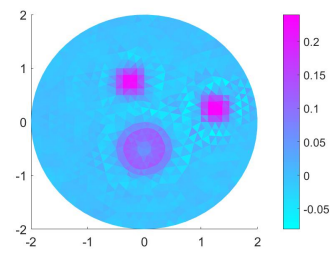

(e) $q_{\mu}$

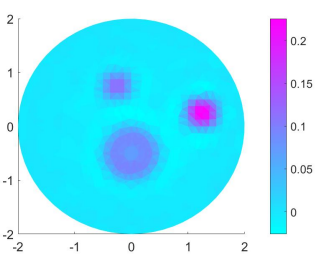

(c) $q_{\rho}$

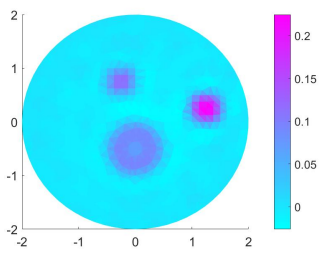

(f) $q_{\rho}$

FIG. 4. Example 1: The reconstruction of perturbed parameters with noise level $\delta=3 \%$ (a)-(c) and $\delta=5 \%(\mathrm{~d})-(\mathrm{f})$.

looks like the stiffness tensor of the background elastic medium, we can always have better reconstruction than when using just a scale one. Our starting pointing is that for an isotropic elastic medium, the two Lamé parameters have some connection since they are both determined by Young's modulus and Poisson's ratio and have no direct relation with density. Thus, we take a similar form of stiffness tensor as the choice of step size, for example, which means that the modification for both $q_{\lambda}, q_{\mu}$ is determined by the combinations of the first two components of $\left(N_{q}^{\prime}\right) *(h)$, and the modification for density $q_{\rho}$ is only determined by the third component of $\left(N_{q}^{\prime}\right) *(h)$. There should be better choices of $\alpha$ than the special one we used.

Example 3. In this example, we consider the reconstruction from phaseless data. The numerical results are shown in Figure 6 and indicate the effectiveness of our 


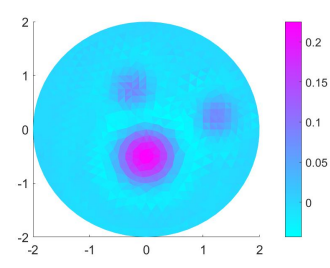

(a) $q_{\lambda}$

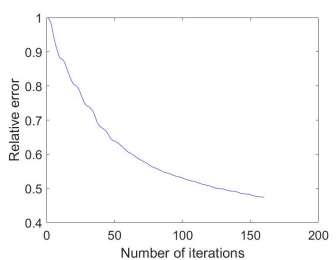

(d) $e_{q_{\lambda}}$

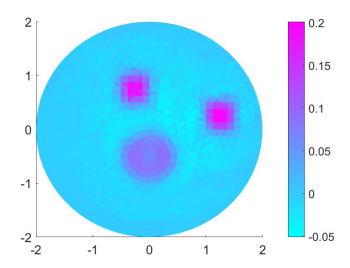

(b) $q_{\mu}$

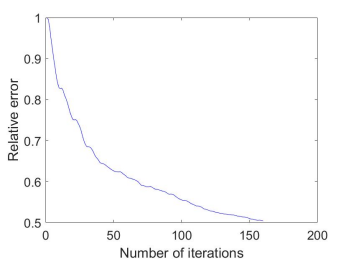

(e) $e_{q_{\mu}}$

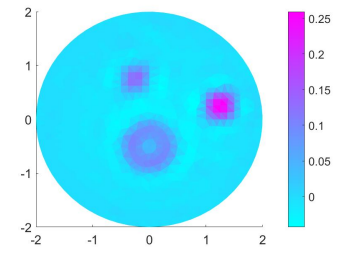

(c) $q_{\rho}$

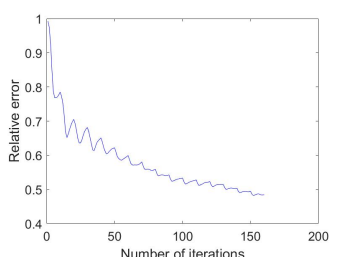

(f) $e_{q_{\rho}}$

FIG. 5. Example 2: The reconstruction of perturbed parameters.

method for the reconstruction from phaseless data.

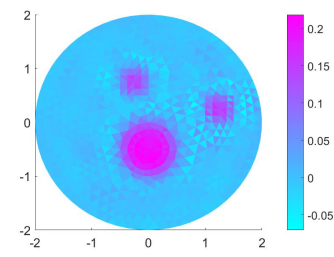

(a) $q_{\lambda}$

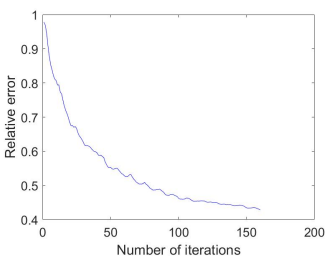

(d) $e_{q_{\lambda}}$

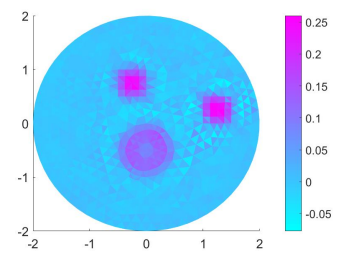

(b) $q_{\mu}$

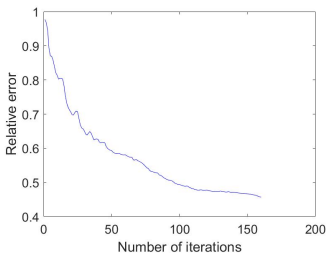

(e) $e_{q_{\mu}}$

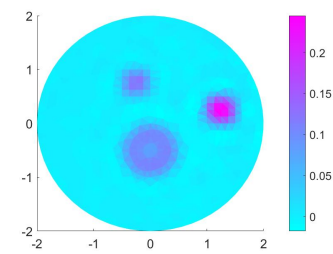

(c) $q_{\rho}$

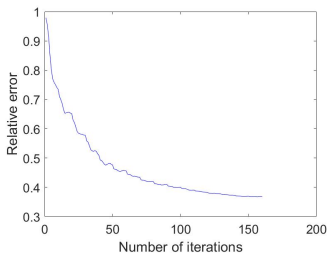

(f) $e_{q_{\rho}}$

FIG. 6. Example 3: The reconstruction of perturbed parameters.

Example 4. We use the measurements generated by the plane pressure incident wave with one fixed direction $d=(1,0)^{\top}$ (i.e., $M=1$ ). In this case the number of iterations at each frequency is set as $L=50$. The reconstruction results and relative errors are shown in Figure 7.

Example 5. In this example, we consider the special case discussed in section 4 . The exact value of $q_{\rho}$ is given by

$$
\begin{aligned}
q_{\rho}= & 0.3\left(1-3 x_{1}\right)^{2} \exp \left(-9 x_{1}^{2}-\left(3 x_{2}+1\right)^{2}\right)-\left(0.6 x_{1}-27 x_{1}^{3}-3^{5} x_{2}^{5}\right) \exp \left(-9 x_{1}^{2}-9 x_{2}^{2}\right) \\
& -0.03 \exp \left(-\left(3 x_{1}+1\right)^{2}-9 x_{2}^{2}\right) ;
\end{aligned}
$$

see Figure 8. Choose $\alpha=0.01$ and $\omega_{\max }=11$. The reconstructed $q_{\rho}$ and relative 


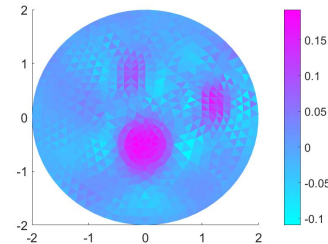

(a) $q_{\lambda}$

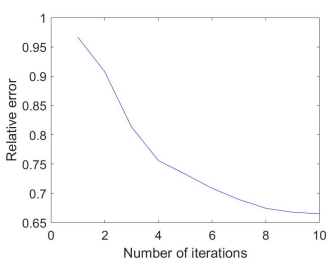

(d) $e_{q_{\lambda}}$

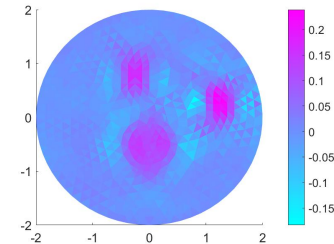

(b) $q_{\mu}$

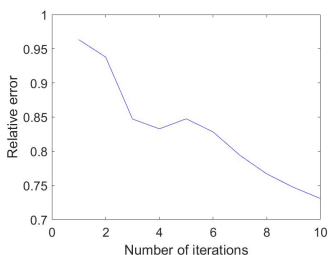

(e) $e_{q_{\mu}}$

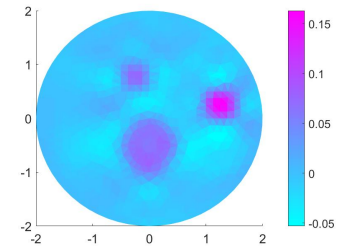

(c) $q_{\rho}$

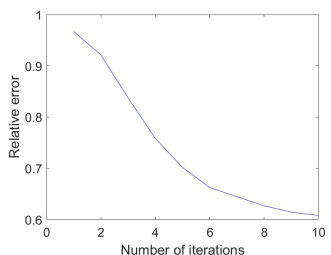

(f) $e_{q_{\rho}}$

Fig. 7. Example 4: The reconstruction of perturbed parameters.

TABLE 2

Final reconstruction errors of $q_{\rho}$ for Examples 5-6.

\begin{tabular}{|c|c|c|}
\hline Figure & Comment & Error of $q_{\rho}$ \\
\hline 9 & Plane pressure incidence & 0.035 \\
\hline 10 & Phaseless measurement & 0.24 \\
\hline $11(\mathrm{a})$ & One small frequency, one $\theta^{i n c}$ & 0.80 \\
\hline $11(\mathrm{~b})$ & One small frequency, multiple $\theta^{i n c}$ & 0.78 \\
\hline $12(\mathrm{a})$ & One large frequency, multiple $\theta^{i n c}$ & 0.14 \\
\hline $12(\mathrm{~b})$ & One $\theta^{i n c}, L=10$ & 0.65 \\
\hline $12(\mathrm{c})$ & One $\theta^{i n c}, L=100$ & 0.59 \\
\hline 13 & Multiple-frequency, one $\theta^{i n c}$ & 0.46 \\
\hline
\end{tabular}

errors from multifrequency measurements with plane pressure incident wave are shown in Figures 9 and 10.

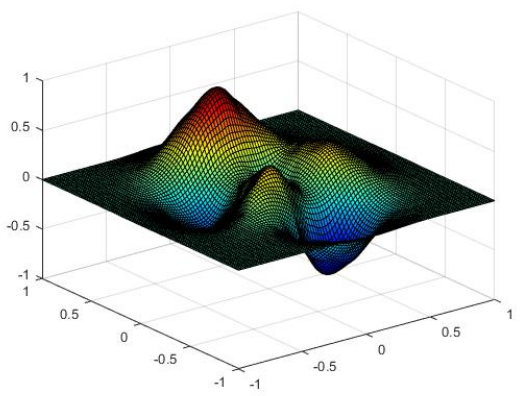

FIG. 8. Example 5: The exact value of $q_{\rho}$.

Example 6. Finally, as a comparison, we consider the reconstruction of mass density only from data at a fixed frequency. For small frequency, it can be seen from Figure 11 that the reconstruction results are extremely bad regardless of whether we have only one or multiple directions of incident waves. But for high frequency 


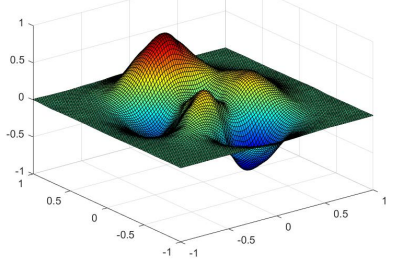

(a) $q_{\rho}$

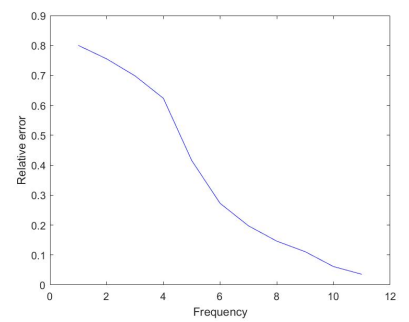

(b) $e_{q_{\rho}}$

FiG. 9. Example 5: The reconstruction of $q_{\rho}$ and relative errors from original measurements.

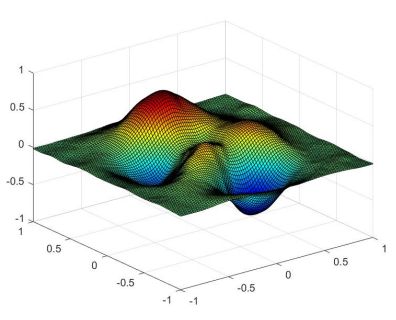

(a) $q_{\rho}$

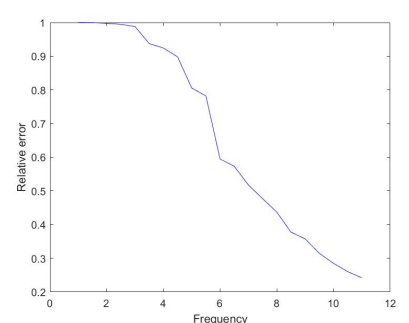

(b) $e_{q_{\rho}}$

FIG. 10. Example 5: The reconstruction of $q_{\rho}$ and relative errors from phaseless measurements.

(see Figure 12(a)), we still can have good reconstruction if we have multiple incident waves. Once we only have one fixed incident wave with direction $d=(0,1)^{\top}$, we cannot obtain good reconstruction results by increasing $L$; see Figure 12((b), (c)). However, by increasing the number of the frequency $(N=11)$, we still can reconstruct some information about $q_{\rho}$; see Figure 13 and the results in Example 4. This further indicates the advantages of taking multifrequency data.

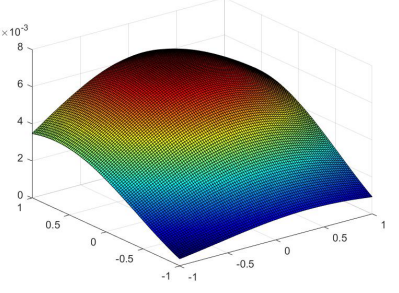

(a) $M=1$

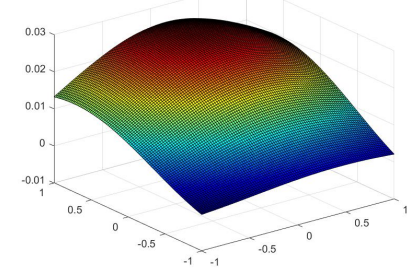

(b) $M=16$

FIG. 11. Example 6: The reconstruction of $q_{\rho}$ from data at $k=1$. 


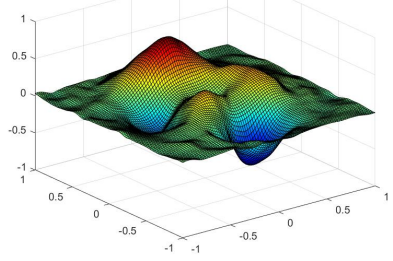

(a) $M=16, L=10$

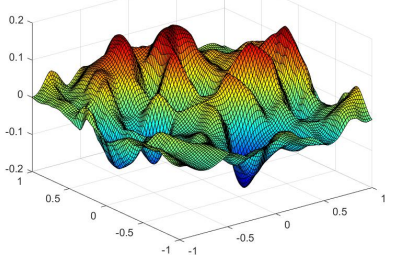

(b) $M=1, L=10$

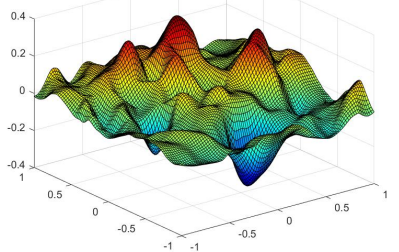

(c) $M=1, L=100$

FIG. 12. Example 6: The reconstruction of $q_{\rho}$ from data at $k=11$.

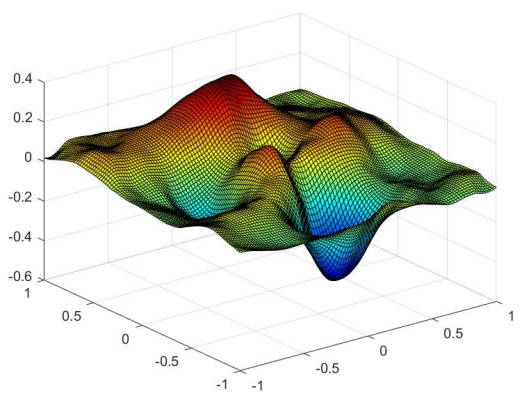

FIG. 13. Example 6: The reconstruction of $q_{\rho}$ from multifrequency data with a fixed incident direction

Copyright $\odot$ by SIAM. Unauthorized reproduction of this article is prohibited. 
We conclude from the above numerical tests that satisfactory reconstructions are obtained through the proposed Landweber iterative algorithms.

Acknowledgments. The authors also would like to thank Prof. Peijun Li for his suggestions on this work. We thank the referees for their constructive comments, which helped to improve the paper.

\section{REFERENCES}

[1] M. Akamatsu, G. Nakamura, and S. Steinberg, Identification of Lamé coefficients from boundary observations, Inverse Problems, 7 (1991), pp. 335-354.

[2] G. Alessandrini, M. di Cristo, A. Morassi, and E. Rosset, Stable determination of an inclusion in an elastic body by boundary measurements, SIAM J. Math. Anal., 46 (2014), pp. 2692-2729, https://doi.org/10.1137/130946307.

[3] H. Ammari, E. Bretin, J. Garnier, H. Kang, H. Lee, and A. Wahab, Mathematical Methods in Elasticity Imaging, Princeton Series in Applied Mathematics, Princeton University Press, Princeton, NJ, 2015.

[4] I. BABušKa AND A. AzIZ, Survey lectures on mathematical foundations of the finite element method, in The Mathematical Foundations of the Finite Element Method with Application to Partial Differential Equations, A. Aziz, ed., Academic Press, New York, 1972, pp. 5-359.

[5] G. BAO, G. Hu, Y. KIAN, AND T. YIN, Inverse source problems in elastodynamics, Inverse Problems, 34 (2018), 045009.

[6] G. BaO, G. Hu, J. Sun, And T. Yin, Direct and inverse elastic scattering from anisotropic media, J. Math. Pures Appl., 117 (2018), pp. 263-301.

[7] G. BAO AND P. LI, Inverse medium scattering for three-dimensional time harmonic Maxwell's equations, Inverse Problems, 20 (2004), pp. L1-L7.

[8] G. BAO AND P. LI, Inverse medium scattering for the Helmholtz equation at fixed frequency, Inverse Problems, 21 (2005), pp. 1621-1641.

[9] G. BAO AND P. LI, Inverse medium scattering problems for electromagnetic waves, SIAM J. Appl. Math., 65 (2005), pp. 2049-2066, https://doi.org/10.1137/040607435.

[10] G. BAO AND P. LI, Inverse medium scattering problems in near-field optics, J. Comput. Math., 25 (2007), pp. 252-265.

[11] G. BAO AND P. LI, Numerical solution of an inverse medium scattering problem for Maxwell's equations at fixed frequency, J. Comput. Phys., 228 (2009), pp. 4638-4648.

[12] G. BAO, P. LI, J. Lin, AND F. TRIKI, Inverse scattering problems with multi-frequencies, Inverse Problems, 31 (2015), 093001.

[13] G. BAO, P. LI, AND Y. ZhaO, Stability in the inverse source problem for elastic and electromagnetic waves with multi-frequencies, J. Math. Pures Appl., to appear; available online at http://www.math.purdue.edu/ lipeijun/paper/accepted/BLZ_JMPA_2019.pdf.

[14] G. BaO, J. Lin, And F. TRIKI, A multi-frequency inverse source problem, J. Differential Equations, 249 (2010), pp. 3443-3465.

[15] G. Bao And F. Triki, Stability Estimates for the One Dimensional Multifrequency Inverse Medium Problem, preprint.

[16] G. BAO AND F. TRIKI, Error estimates for the recursive linearization of inverse medium problems, J. Comput. Math., 28 (2010), pp. 725-744.

[17] E. Beretta, M. V. de Hoop, E. Francini, S. Vessella, and J. Zhai, Uniqueness and Lipschitz stability of an inverse boundary value problem for time-harmonic elastic waves, Inverse Problems, 33 (2017), 035013.

[18] E. Beretta, E. Francini, and S. Vessella, Uniqueness and Lipschitz stability for the identification of Lamé parameters from boundary measurements, Inverse Problems Imaging, 8 (2014), pp. 611-644.

[19] J. Cheng, V. IsAKov, AND S. Lu, Increasing stability in the inverse source problem with many frequencies, J. Differential Equations, 260 (2016), pp. 4786-4804.

[20] P. G. Ciarlet, Mathematical Elasticity, Vol. I. Three-Dimensional Elasticity, North-Holland, Amsterdam, 1988.

[21] G. Eskin And J. Ralston, On the inverse boundary value problem for linear isotropic elasticity, Inverse Problems, 18 (2002), pp. 907-922.

[22] D. Givoli And J. Keller, Non-reflecting boundary conditions for elastic waves, Wave Motion, 12 (1990), pp. 261-279.

[23] M. Hanke, A. Neubauer, And O. Scherzer, A convergence analysis of the Landweber iteration for nonlinear ill-posed problems, Numer. Math., 72 (1995), pp. 21-37.

Copyright $@$ ( ) by SIAM. Unauthorized reproduction of this article is prohibited. 
[24] G. Hsiao, R. Kleinman, and G. Roach, Weak solutions of fluid-solid interaction problems, Math. Nachr., 218 (2000), pp. 139-163.

[25] G. Hu, P. Li, X. LiU, And Y. ZhaO, Inverse source problems in electrodynamics, Inverse Problems Imaging, 12 (2018), pp. 1411-1428.

[26] M. Ikenata, Inversion formulas for the linearized problem for an inverse boundary value problem in elastic prospection, SIAM J. Appl. Math., 50 (1990), pp. 1635-1644, https: //doi.org/10.1137/0150097.

[27] O. Imanuvilov and M. Yamamoto, Global uniqueness in inverse boundary value problems for the Navier-Stokes equations and Lamé system in two dimensions, Inverse Problems, 31 (2015), 035004.

[28] V. ISAKOV AND S. Lu, Increasing stability in the inverse source problem with attenuation and many frequencies, SIAM J. Appl. Math., 78 (2018), pp. 1-18, https://doi.org/10.1137/ $17 \mathrm{M} 1112704$.

[29] B. Kaltenbacher, A. Neubauer, and O. Scherzer, Iterative Regularization Methods for Nonlinear Ill-Posed Problems, Walter de Gruyter, Berlin, 2008.

[30] V. D. Kupradze, T. G. Gegelia, M. O. Basheleǐshvili, and T. V. Burchuladze, ThreeDimensional Problems of the Mathematical Theory of Elasticity and Thermoelasticity, North-Holland, Amsterdam, 1979.

[31] P. Li, Y. WANG, Z. WANG, AND Y. ZHAO, Inverse obstacle scattering for elastic waves, Inverse Problems, 32 (2016), 115018.

[32] P. Li AND G. YUAN, Increasing stability for the inverse source scattering problem with multifrequencies, Inverse Problems Imaging, 11 (2017), pp. 745-759.

[33] P. Li AND X. YUan, Inverse obstacle scattering for elastic waves in three dimensions, Inverse Problems Imaging, 13 (2019), pp. 545-573.

[34] G. Nakamura and G. Uhlmann, Identification of Lamé parameters by boundary measurements, Amer. J. Math., 115 (1993), pp. 1161-1187.

[35] G. Nakamura and G. Uhlmann, Global uniqueness for an inverse boundary problem arising in elasticity, Invent. Math., 118 (1994), pp. 457-474.

[36] G. NAKAmura AND G. Uhlmann, Inverse problems at the boundary for an elastic medium, SIAM J. Math. Anal., 26 (1995), pp. 263-279, https://doi.org/10.1137/ S0036141093247494.

Copyright $@$ by SIAM. Unauthorized reproduction of this article is prohibited. 